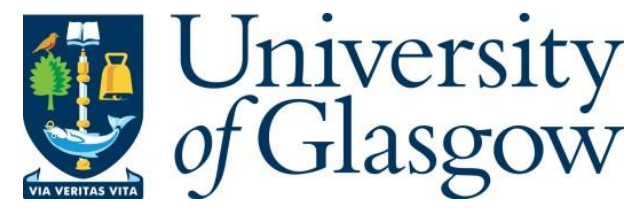

Ghorai, S., and Hill, N.A. (1999) Development and stability of gyrotactic plumes in bioconvection. Journal of Fluid Mechanics, 400 . pp. 1-31. ISSN 0022-1120 (doi:10.1017/S0022112099006473)

Copyright @ 1999 Cambridge University Press

A copy can be downloaded for personal non-commercial research or study, without prior permission or charge

Content must not be changed in any way or reproduced in any format or medium without the formal permission of the copyright holder(s)

When referring to this work, full bibliographic details must be given

http://eprints.gla.ac.uk/88795/

Deposited on: 20 December 2013

Enlighten - Research publications by members of the University of Glasgow http://eprints.gla.ac.uk 


\title{
Development and stability of gyrotactic plumes in bioconvection
}

\author{
By S. GHORAI AND N. A. HILL \\ Department of Applied Mathematics, University of Leeds, Leeds LS2 9JT, UK
}

(Received 23 January 1998 and in revised form 12 March 1999)

Using the continuum model of Pedley, Hill \& Kessler (1988) for bioconvection in a suspension of swimming, gyrotactic micro-organisms, we investigate the existence and stability of a two-dimensional plume in tall, narrow chambers with stress-free sidewalls. The system is governed by the Navier-Stokes equations for an incompressible fluid coupled with a micro-organism conservation equation. These equations are solved numerically using a conservative finite-difference scheme. In sufficiently deep chambers, the plume is always unstable to both varicose and meandering modes. A linear stability analysis for an infinitely long plume predicts the growth rates of these instabilities, explains the mechanisms, and is in good agreement with the numerical results.

\section{Introduction}

Bioconvection is the name given to pattern formation in suspensions of microorganisms, such as bacteria and algae, due to up-swimming of the micro-organisms (Pedley \& Kessler 1992). In all cases the micro-organisms are denser than water and on average they swim upwards (although the reasons for up-swimming may be different for different species). The algae (e.g. Chlamydomonas) are approximately $5 \%$ denser than water, whereas the bacteria are nearly $10 \%$ denser than water. Microorganisms respond to certain stimuli by tending to swim in particular directions. These responses are called taxes, examples being gravitaxis, phototaxis, chemotaxis and gyrotaxis. Gravitaxis indicates swimming in the opposite sense to gravity, phototaxis denotes swimming towards or away from light, and chemotaxis corresponds to swimming up chemical gradients. Gyrotaxis is swimming directed by the balance between the torque due to gravity acting on a bottom-heavy cell and the torque due to viscous forces arising from local shear flows. This paper is concerned with gyrotaxis.

The first detailed observations of bioconvection were carried out at the beginning of this century by Wager (1911) but the subject was not taken up again until the work of Platt (1961), who apparently coined the term 'bioconvection'. Plesset \& Winet (1974) made some measurements of the wavelengths of the bioconvection patterns at the onset of instability in a suspension of the ciliate, Tetrahymena pyriformis, which is negatively gravitactic (but not apparently gyrotactic (Kessler 1985a)) and showed agreement with the linear stability theory for a layer of dense fluid overlying a layer of light fluid. Kessler (1985a) demonstrated that many swimming micro-organisms are gyrotactic and made observations (Kessler 1985b) of both the almost regular patterns that occur in concentrated algal suspensions in shallow layers a few millimetres deep, and of gyrotactic plume formation in a tall narrow cylindrical tube. Figure $2(d)$ in the 
review by Pedley \& Kessler (1992) shows irregular tall thin bottom-standing plumes several centimetres deep and their instabilities which are modelled in this paper, but there have not yet been any quantitative studies of these plumes. A recent quantitative study of bioconvection in algal suspensions was conducted by Bees \& Hill (1997), who measured the wavelengths of the planforms of shallow bioconvection patterns as a function of the depth and concentration of the suspension. Hill \& Häder (1997) measured the trajectories of individual swimming algal cells and showed that their motion is well-characterized by the limit of a correlated biased random walk in which the time step tends to zero. They were able to calculate the statistical moments required for the coefficients of the Fokker-Planck equation for the cells' orientational probability density function. These coefficients are needed in Pedley \& Kessler's (1990) new continuum model.

The centre of mass of a typical gyrotactic cell is displaced from the centre of buoyancy so that in the absence of any flow it swims vertically upwards (gravitaxis). Due to up-swimming, the top layer of the suspension becomes denser than the layer below. If the parameters (e.g. the Rayleigh number) are above critical values, this leads to a convective instability and formation of convection patterns. This phenomenon is known as 'bioconvection'; it has some similarity with Rayleigh-Bénard convection, but is driven solely by the swimming of the micro-organisms. Pedley, Hill \& Kessler (1988) extended the theory of Childress, Levandowsky \& Spiegel (1975) (who analysed the bioconvection instability of a suspension of purely gravitactic cells) to develop a continuum model for a suspension of gyrotactic micro-organisms.

Micro-organisms are of various shapes and sizes. For simplicity, algal cells such as Chlamydomonas (whose shapes closely approximate a spheroid) are idealized here as spheres of radius $a$. Figure 1 shows such a cell placed in a shear flow. The force of gravity, acting through the centre of mass exerts a torque $\boldsymbol{T}_{g}=m \boldsymbol{h} \times \boldsymbol{g}$, where $m$ is the mass of the cell and $\boldsymbol{g}$ is the acceleration due to gravity. For algal cells, $h$ is a few percent of the cell radius. The viscous torque on a sphere of radius $a$ in a fluid of viscosity $\mu$ is given by

$$
\boldsymbol{T}_{\mu}=4 \pi \mu a^{3}(\nabla \times \boldsymbol{u}-2 \boldsymbol{\Omega}),
$$

where $\boldsymbol{u}$ is the fluid velocity field and $\boldsymbol{\Omega}$ is the sphere's angular velocity. The rate-ofstrain tensor gives rise to an additional torque only on aspherical bodies (Batchelor 1970). Hence the total torque is given by

$$
\boldsymbol{T}=4 \pi \mu a^{3}(\nabla \times \boldsymbol{u}-2 \boldsymbol{\Omega})+m \boldsymbol{h} \times \boldsymbol{g} .
$$

Since inertial effects are negligible at the low Reynolds numbers associated with the motion of individual cells, their orientation is specified by $\boldsymbol{T}=0$ so that

$$
\frac{\mathrm{d} \theta}{\mathrm{d} t}=-\frac{\sin \theta-B \zeta}{2 B},
$$

where $\zeta$ is the horizontal component of the vorticity and $B=4 \pi \mu a^{3} / m g h$ is the time scale for the reorientation of the micro-organisms by the gravitational torque against viscous resistance and is called the 'gyrotactic orientation parameter' by Pedley \& Kessler (1987).

Thus gravity and the vorticity can orient individual cells and guide their trajectories. Gyrotaxis can be demonstrated in an experiment in a slow Poiseuille flow down a vertical tube of circular cross-section. The balance between gravitational and viscous torques gives one stable equilibrium orientation with individual cells tipped away from the upward vertical towards the axis of the pipe. The cells swim towards the axis as 


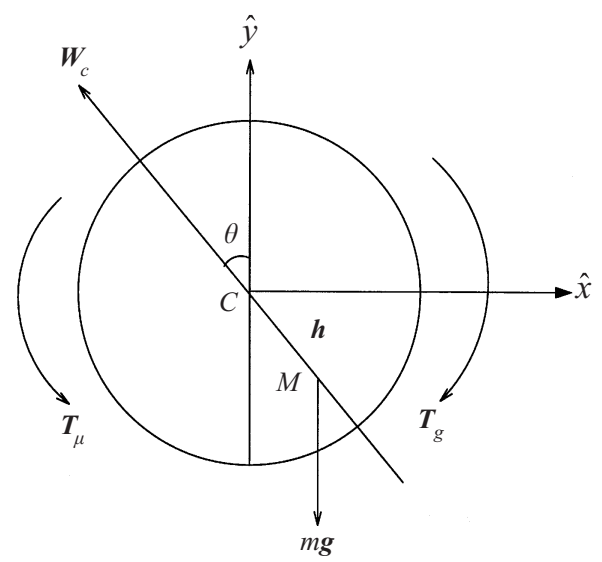

FIGURE 1. An idealized algal cell. $\theta$ increases in the anti-clockwise sense and $\boldsymbol{h}$ denotes the displacement of the centre of gravity from the centre of the cell. $\boldsymbol{h}=h(\hat{\boldsymbol{x}} \sin \theta-\hat{\boldsymbol{y}} \cos \theta)$ relative to Cartesian coordinates, $C x y$, with the origin at the centre of the cell, $C$, and the horizontal, $x$, and the vertical, $y$, directions fixed relative to the laboratory. $M$ is the centre of mass and $\boldsymbol{W}_{c}$ is the swimming velocity of the micro-organisms relative to the water.

they are carried along in the pipe flow, and focus into a narrow beam. Conversely, if the direction of the flow is reversed, the cells are oriented away from the axis toward the walls, confirming the role of gyrotaxis in cell orientation.

Gravity also enters in another, entirely different manner in producing co-operative phenomena (Kessler 1985b). The local average fluid density is modified by the presence of cells. If there is a small region with a greater than average concentration of cells, the excess density is sufficient to produce a substantial sinking velocity. The sinking region produces a fluid velocity field which guides further gyrotactic accumulation perpendicular to it. This positive feedback generates and maintains the sharply focused descending plumes of cells frequently observed in dense algal cultures. The focused beam of algae is often observed to develop an instability in the form of regularlyspaced axisymmetric 'blobs' (Kessler 1985b). The blobs are regions of increased cell concentration, which are wider than the beam. They fall faster than the centreline velocity and therefore have an internal vortex-ring structure.

Harashima, Watanabe \& Fujishiro (1988) solved the equations of bioconvection numerically for purely up-swimming cells (i.e. $\theta=0$ ) in a two-dimensional layer of finite depth and width, and studied the evolution of bioconvection from an initially uniform state. They proposed minimum potential energy as a principle for the determination of the steady-state roll size for a given value of Rayleigh number and a given box size. Their computational domain had a width/height ratio of 8 . In contrast, we consider numerical solutions of the equations of bioconvection for gyrotactic cells in deep chambers using the continuum model of Pedley et al. (1988).

Timm \& Okubo (1994) derived a steady-state axisymmetric plume model for gyrotactic cells but some of their assumptions are unlikely to be valid. In particular, the horizontal component of swimming due to gyrotaxis was taken to be proportional to the vertical fluid velocity, even though the torque balance equation shows it to be proportional to the gradient of the fluid velocity. Also, the fluid viscosity and diffusion due to the cells were neglected without justification. (Indeed, we show that the dominant balance in the cell concentration profile in a plume is between the horizontal components of gyrotaxis and cell diffusion.) Pedley (1988) tried to 
construct a model of 'bottom-standing plumes' for a two-dimensional horizontally periodic pattern and derived estimates for the characteristic scalings but was unable to obtain a fully consistent solution. In this paper, we shall derive a steady-state plume model for a given plume spacing, assuming only that the solutions are independent of the vertical coordinate. This is consistent with the numerical experiments.

We shall study two-dimensional bioconvection in a layer confined by a rigid bottom, a stress-free top and lateral boundaries in the nonlinear regime. A wider chamber will generate several plumes that are periodic in $x$ with vertical stress-free boundaries between them. The aim of the present computation is to study the structure and stability of just one of them. Since we specify the plume spacing in advance, the present study will not shed light on the wavenumber selection process. This paper is organized as follows. The mathematical formulation of the continuum model is outlined in $\S 2$. In $\S 3$, a brief description of the computational method is given, and a comparison of the numerical results with the linear stability results of Hill, Pedley \& Kessler (1989) is given in $\S 4$. The results of the numerical computations are presented in $\S 5$. We analyse the stability of an infinitely long gyrotactic plume in $\S 6$ and, in $\S 7$, we make comparisons with the numerical results and draw conclusions.

\section{Mathematical formulation}

The geometry considered consists of a two-dimensional rectangular box of width $L$ and height $H$ referred to Cartesian coordinates with the $y$-axis pointing upwards. The left, right and top walls of the chamber are stress free and the bottom wall is rigid. There is no flux of cells through any of the walls.

\subsection{Governing equations}

As in Pedley et al. (1988), we assume a monodisperse cell population which can be modelled by a continuous distribution. The suspension is dilute so that the volume fraction of the cells is small and cell-cell interactions are negligible. Each cell has a volume $\vartheta$ and density $\rho+\Delta \rho$, where $\rho$ is the density of the water in which the cells swim and $\Delta \rho / \rho \ll 1$. The velocity $\boldsymbol{u}$ is solenoidal and the vorticity, $\boldsymbol{\omega}=\operatorname{curl} \boldsymbol{u}=(0,0, \zeta)$; thus we introduce a stream function $\psi$ such that

$$
\boldsymbol{u}=(u, v, 0)=\left(\frac{\partial \psi}{\partial y},-\frac{\partial \psi}{\partial x}, 0\right), \quad \zeta=-\nabla^{2} \psi .
$$

Conservation of cells requires that the number of cells per unit volume, $n$, satisfies the equation

$$
\frac{\partial n}{\partial t}=-\nabla \cdot J
$$

where the flux of cells is

$$
\boldsymbol{J}=n \boldsymbol{u}+n W_{c} \overline{\boldsymbol{p}}-D \nabla n .
$$

The third term on the right-hand side of (5) represents the random component of cell locomotion. We assume that the diffusion coefficient $D$ is homogeneous and isotropic and independent of the other parameters of the problem. The second term in (5) arises due to the average swimming of the cells: $W_{c} \overline{\boldsymbol{p}}$ is the average swimming velocity relative to the fluid and $W_{c}$ is assumed to be constant; $\overline{\boldsymbol{p}}(\boldsymbol{x}, t)$ represents the average orientation of cells. 
The vorticity evolves according to the equation

$$
\frac{\partial \zeta}{\partial t}+\nabla \cdot(\zeta \boldsymbol{u})=v \nabla^{2} \zeta-\frac{\Delta \rho g \vartheta}{\rho} \frac{\partial n}{\partial x}
$$

Here $v$ is the kinematic viscosity and (6) is derived under the Boussinesq approximation, neglecting all effects of the cells on the fluid, except their negative buoyancy, because the suspension is dilute.

\subsection{Calculation of the mean direction}

From figure 1, we have

$$
\boldsymbol{p} \equiv\left(p_{x}, p_{y}\right)=(-\sin \theta, \cos \theta),
$$

where $\theta$ is the solution of (2). If the shear is sufficiently small so that $|B \zeta| \leqslant 1$, then the steady-state orientation is obtained by setting the left-hand side of (2) equal to zero. Thus when $|B \zeta| \leqslant 1$, we find

$$
\overline{\boldsymbol{p}}=\left(-\kappa,\left(1-\kappa^{2}\right)^{1 / 2}\right), \quad|\kappa| \leqslant 1,
$$

where $\kappa=B \zeta$. If the vorticity is large $(|B \zeta|>1)$, the cell tumbles but swims on average in a fixed direction at an angle to the vertical (Kessler 1985b). When the vorticity is large, the swimming direction $\boldsymbol{p}$ is approximated in the following way.

First consider the case when $\kappa=B \zeta>1$. Define $\tau \equiv t / 2 B$, then (2) becomes

$$
\frac{\mathrm{d} \theta}{\mathrm{d} \tau}=\kappa-\sin \theta,
$$

which gives

$$
\kappa \tan \left(\frac{\theta}{2}\right)-1=\gamma \tan \left\{\frac{\gamma(\tau+C)}{2}\right\},
$$

where $\gamma=\left(\kappa^{2}-1\right)^{1 / 2}$ and $C$ is a constant of integration.

To find the period of rotation in terms of $\tau$, note that as $\theta$ increases from 0 to $2 \pi$, $\theta / 2$ increases by $\pi$ and thus $\gamma(\tau+C) / 2$ changes by $\pi$, which implies that the period is $T=2 \pi / \gamma$.

Let $\overline{\boldsymbol{p}}=\int_{0}^{T} \boldsymbol{p} \mathrm{d} \tau / T$ be the average value of $\boldsymbol{p}$ over a period, thus

$$
\bar{p}_{x}=\frac{1}{T} \int_{0}^{2 \pi} \frac{-\sin \theta}{\mathrm{d} \theta / \mathrm{d} \tau} \mathrm{d} \theta=-\kappa+\gamma,
$$

and

$$
\bar{p}_{y}=\frac{1}{T} \int_{0}^{2 \pi} \frac{\cos \theta}{\kappa-\sin \theta} \mathrm{d} \theta=0,
$$

which is expected since the horizontal line through the centre of the cell is a line of symmetry for the rotation of the cell. Thus, if $\kappa=B \zeta>1$, then

$$
\overline{\boldsymbol{p}}=\left(-\kappa+\left(\kappa^{2}-1\right)^{1 / 2}, 0\right)
$$

and similarly, if $\kappa=B \zeta<-1$, then

$$
\overline{\boldsymbol{p}}=\left(-\kappa-\left(\kappa^{2}-1\right)^{1 / 2}, 0\right) .
$$

Equations (7), (9) and (10) determine the average swimming direction of the cells for any value of $\zeta$. 
The equations are scaled using the width $L$, the time scale $L^{2} / D$ and the mean concentration $\bar{n}$. The resulting system of coupled equations is

$$
\begin{gathered}
\boldsymbol{u}=(u, v, 0)=\left(\frac{\partial \psi}{\partial y},-\frac{\partial \psi}{\partial x}, 0\right), \quad \zeta=-\nabla^{2} \psi, \\
\frac{\partial \zeta}{\partial t}+\nabla \cdot(\zeta \boldsymbol{u})=S_{c} \nabla^{2} \zeta-S_{c} R \frac{\partial n}{\partial x},
\end{gathered}
$$

and

$$
\frac{\partial n}{\partial t}=-\nabla \cdot J
$$

where the flux of cells is

$$
\boldsymbol{J}=n \boldsymbol{u}+n V_{c} \overline{\boldsymbol{p}}-\nabla n .
$$

For convenience we have kept the same notation for the dimensional and dimensionless variables. Here $S_{c}=v / D$ is the Schmidt number, $V_{c}=W_{c} L / D$ is the scaled cell swimming speed and $R$ is a Rayleigh number defined as

$$
R=\frac{\bar{n} \vartheta \Delta \rho g L^{3}}{\rho v D} ;
$$

$\overline{\boldsymbol{p}}$ is defined by

$$
\overline{\boldsymbol{p}}= \begin{cases}\left(-\kappa,\left(1-\kappa^{2}\right)^{1 / 2}\right), & |\kappa| \leqslant 1, \\ \left(\left[-|\kappa|+\left(\kappa^{2}-1\right)^{1 / 2}\right] \frac{\kappa}{|\kappa|}, 0\right), & |\kappa|>1,\end{cases}
$$

where $\kappa=G \zeta$ and $G=B D / L^{2}$ is defined as the gyrotaxis number. The boundary conditions are applied at

$$
x= \pm \frac{1}{2} \text { and } y=0, \lambda,
$$

where $\lambda=H / L$ is the aspect ratio of the chamber.

The definition of $R$ is non-standard in that it is based on the width and mean cell concentration rather than the height and cell concentration at the top of the chamber. The conventional Rayleigh number increases with an increase in the height of the chamber, whereas our Rayleigh number remains constant. We use our definition so that the height of the chamber can be varied independently of the other parameters. $R V_{c} \lambda^{4} /\left(1-\exp \left(-V_{c} \lambda\right)\right)$ equals the conventional Rayleigh number at a given aspect ratio. Note also that the bioconvection equations reduce to those of the thermal convection when $V_{c}=0$.

\subsection{Initial and boundary conditions}

We impose rigid, no-slip boundary conditions at the bottom wall and assume that the other boundaries are stress-free, so that

$$
\left.\begin{array}{c}
\psi=0 \quad \text { at } x= \pm \frac{1}{2} \text { and } y=0, \lambda, \\
\frac{\partial \psi}{\partial y}=0 \quad \text { at } y=0, \quad \frac{\partial^{2} \psi}{\partial x^{2}}=0 \quad \text { at } x= \pm \frac{1}{2}, \quad \frac{\partial^{2} \psi}{\partial y^{2}}=0 \quad \text { at } y=\lambda .
\end{array}\right\}
$$

The boundary condition on $n$ is that there be no flux of cells through the walls, thus

$$
\boldsymbol{J} \cdot \hat{\boldsymbol{x}}=0 \quad \text { at } x= \pm \frac{1}{2} \quad \text { and } \quad \boldsymbol{J} \cdot \hat{\boldsymbol{y}}=0 \quad \text { at } y=0, \lambda .
$$


The initial conditions are that of a uniform state together with a small perturbation to the uniform concentration of cells:

$$
\psi=0, \quad \zeta=0, \quad n=1+\epsilon \cos (m \pi x),
$$

where $\epsilon=10^{-5}$ and $m=2$. The perturbation is applied for computational convenience to ensure that the plume forms in the middle of the chamber.

\section{Numerical procedure}

The governing equations (11)-(13) are discretized using a conservative finitedifference scheme (Ghorai 1997) on a staggered mesh, with the stream function and vorticity stored on one set of nodes and the concentration stored on another set of nodes. The grid is chosen so that the concentration nodes lie in the interior only whereas those of the stream function and vorticity lie in the interior and on the boundary of the domain. The advantage of the staggered mesh is that the no-cell-flux boundary condition can be satisfied immediately when discretized without further approximation. We know that a plume is concentrated along a narrow column and that there are boundary layers at the top and bottom walls due to the large cell concentration and the presence of the rigid wall respectively. In order to describe these gradients accurately, it is necessary to use a non-uniform coordinate mesh. We transform the non-uniform spatial increments $\Delta x_{i}$ and $\Delta y_{j}$ in the finite-difference equations to a uniform grid using an orthogonal transformation $\xi_{k}=\xi_{k}\left(x_{k}\right)$ which also maps the problem to the computational domain $-1 \leqslant \xi_{k} \leqslant 1$, where $x_{k}=(x, y)$ and $\xi_{k}=(\xi, \eta)$. An accurate transformed finite-difference equation for the first derivative $f_{i}^{I}$ is given by

$$
\frac{f_{i+1}-f_{i-1}}{2 \Delta \xi(\mathrm{d} x / \mathrm{d} \xi)_{i}}=f_{i}^{I}\left[1+\frac{(\Delta \xi)^{2}}{6}\left(\frac{\mathrm{d}^{3} x}{\mathrm{~d} \xi^{3}}\right)_{i} /\left(\frac{\mathrm{d} x}{\mathrm{~d} \xi}\right)_{i}\right]
$$

(de Rivas 1972). The second term inside the bracket on the right-hand side of (19) is important only when there is a large grid variation. The second derivative of $f$ is obtained by recursion of (19). The above difference approximation has a truncation error $O\left(\Delta \xi^{2}\right)$ for an arbitrary mesh transformation in problems of boundary layer character. Here we have taken the transformations proposed by Roberts (1970).

An expression for the vorticity boundary condition can be obtained by expanding the stream function near the rigid surface using a three-term Taylor series expansion and the no-slip condition:

$$
\zeta_{\mathrm{w}}=-\frac{\zeta_{\mathrm{nw}}}{2}-\frac{3 \psi_{\mathrm{nw}}}{(\Delta n)^{2}}
$$

where $\zeta_{\mathrm{nw}}, \psi_{\mathrm{nw}}$ are the values of $\psi, \zeta$ at the near-wall node (adjacent to the wall) and $\Delta n$ is the non-dimensional distance of the near-wall node from the wall.

An implicit scheme with Euler backward differencing in time and central differencing in space is used to obtain the transient solutions. A line-by-line tridiagonal matrix algorithm with relaxation is used to solve the nonlinear discretized equations. The number of grid points along the $x$-direction is 36 and the number of grid points along the $y$-direction is increased with an increase in aspect ratio. For example 36 points are taken for $\lambda=1$ and 56 points are taken for $\lambda=4$ along the vertical direction. Some of the results were run with different numbers of grid points to check the grid independence of the solutions. In $\S 5.1$, we compare solutions of different grids for an aspect ratio of 1 . Many of the details of the numerical procedure, and its validation by comparison with a benchmark computation of thermal convection (de Vahl Davis 
1983), are available on request from JFM Editorial office. Full details can be found in the thesis of Ghorai (1997).

\section{Linear stability of the basic equilibrium state}

Equations (11)-(13) possess a steady solution in which $\psi=\zeta=0$ and the concentration has the equilibrium exponential profile

$$
n_{p}(y)=\frac{V_{c} \lambda \exp \left(V_{c} y\right)}{\exp \left(V_{c} \lambda\right)-1} .
$$

HPK have examined the linear stability of this equilibrium solution in a finite-depth chamber. In the absence of gyrotaxis $(G=0)$, the wavelength $(\Lambda)$ of the most unstable mode is large (as found by Childress et al. 1975). On the other hand, in the presence of gyrotaxis, the most unstable wavelength is finite. We compute the critical Rayleigh number as a function of the wavelength using the present code and compare the results with those of Hill et al. (1989). The depth of the chamber is taken as the length scale in this section only. The top and the bottom walls are rigid and the sidewalls are rigid and stress free. The Rayleigh number is

$$
R^{*}=\frac{\bar{n} \vartheta \Delta \rho g H^{3}}{\rho v D},
$$

which is related to the Rayleigh number $R_{H P K}$ of Hill et al. by

$$
R_{H P K}=\frac{R^{*} V_{c}^{2}}{1-\exp \left(-V_{c}\right)} .
$$

The numerical solutions were obtained in the following way. First a solution for a single convection cell was established. This solution is one half of the periodic solution of wavelength equal to twice the chamber width. Then the value of the Rayleigh number was decreased by $5 \%$ and the new solution was allowed to settle to its steady state step by step. The critical Rayleigh number was taken to be approximately the value at which the final steady state is first equal to the equilibrium steady state. The exact Rayleigh number lies within $\pm 5 \%$ of the computed values. Figure 2 shows the neutral curves for $V_{c}=5$ and the agreement between the linear stability analysis and the numerical results is satisfactory. It is difficult to compute the critical Rayleigh number for $G=0.02$ when the wavelength is large $(\Lambda \geqslant 3)$, since the wavelength of the most unstable mode is approximately 1.5 and this mode dominates the numerical solutions in wider chambers.

\section{Results}

To study the effect of the depth on plume formation, the governing parameters $R, S_{c}, G, V_{c}$ are kept constant and the aspect ratio is varied from small to large values. The following parameter values are based on a chamber width of approximately $0.5 \mathrm{~cm}$ using data from table 1 of Kessler (1986) for a suspension of Chlamydomonas nivalis:

$$
R=500, \quad S_{c}=20, \quad G=0.01, \quad V_{c}=10 .
$$

5.1. Aspect ratio $\lambda=1$

The critical Rayleigh number for $S_{c}=20, G=0.01, V_{c}=10$ and $\lambda=1$ is approximately 260 . Since the governing parameters are above the critical values, the 


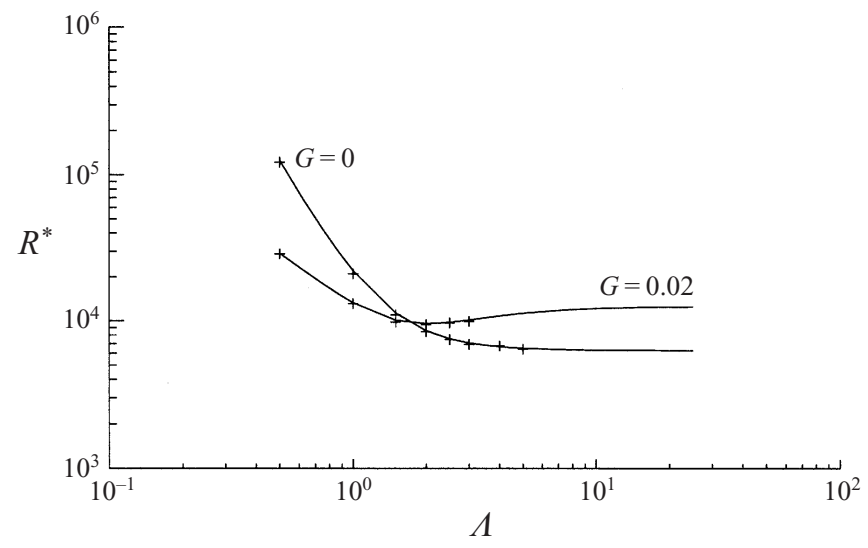

FIGURE 2. Comparison between numerical estimates $(+)$ and the solutions of the linear stability analysis (solid lines) for the neutral curves at the onset of bioconvection when $V_{c}=5$ and $G=0.0$ and 0.02 .

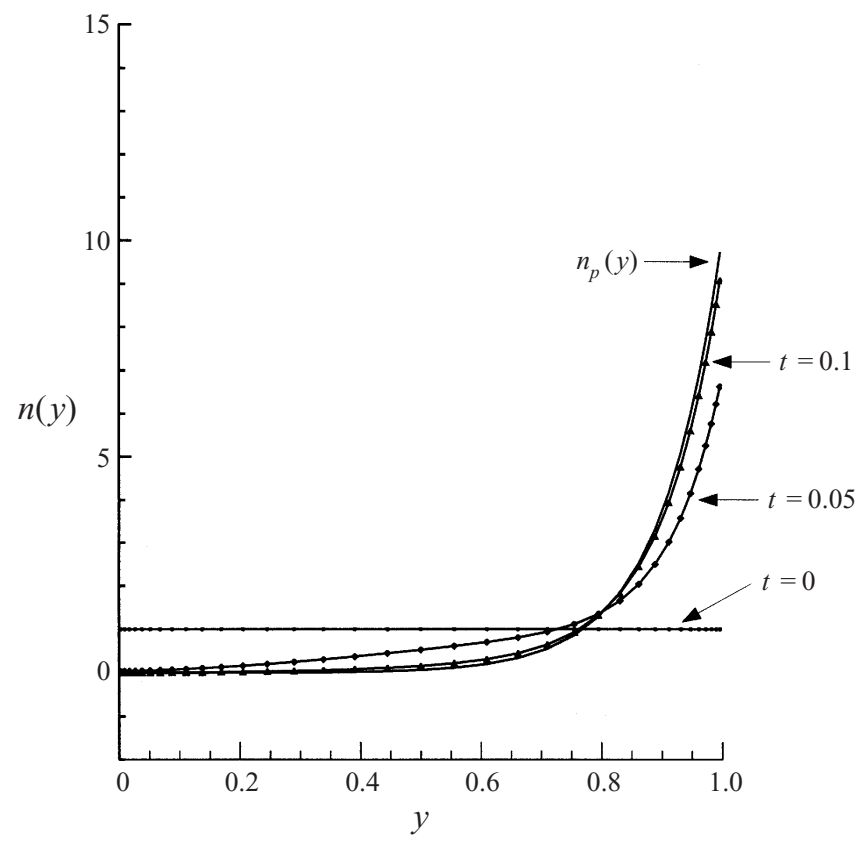

FIGURE 3. Evolution of the cell concentration, $n(y)$, in the mid-vertical plane, for an ultimately unstable suspension at an early stage for aspect ratio $\lambda=1$. The values of the other parameters are given in $(22) . n_{p}(y)$ is the equilibrium profile.

concentration profile initially develops towards (21), but bioconvection begins before the profile develops fully.

Figure 3 shows an example of the initial evolution of cell concentration in the $y$-direction with time and also the solution given by (21). At $t=0$, the concentration is uniform and $n=1$ approximately. The concentration at the top (bottom) of the chamber increases (decreases) with time due to cells swimming upwards and accumulating at the top, where the no-cell-flux condition holds. As time increases, the concentration profile tends to the exponential profile given by (21). Since the 


\begin{tabular}{ccccc} 
Grid & Nodes & $n_{\text {mid }}$ & $\begin{array}{c}\psi_{\max } \\
(x, y)\end{array}$ & $\begin{array}{c}\zeta_{\max } \\
x(y=0)\end{array}$ \\
Stretched & $26 \times 26$ & 3.713 & 1.891 & 56.392 \\
& & & $(0.224,0.583)$ & -0.232 \\
Stretched & $36 \times 36$ & 3.685 & 1.883 & 55.895 \\
& & & $(0.225,0.585)$ & -0.233 \\
Uniform & $46 \times 46$ & \multirow{2}{*}{3.679} & 1.880 & 55.988 \\
& & & $(0.225,0.584)$ & -0.233
\end{tabular}

TABLE 1. Properties of the solution of the final steady state $\left(2^{+}\right)$using different grids when $\lambda=1$, $R=500, S_{c}=20, V_{c}=10$ and $G=10^{-2}$.

parameters are above the critical values, a plume begins to form (solutions become functions of $x$ also) before the equilibrium exponential profile is achieved.

Figure 4 shows the evolution of the plume at various times from the uniform state (18). In these figures, we have plotted $(a)$ cell concentration as a surface, $(b)$ total cell flux vector $\boldsymbol{J}$, and $(c)$ streamline contours. At the beginning, the cells swim upwards and an exponential cell concentration profile begins to develop. There is also a very weak circulation due to the perturbation given to the initial uniform concentration. Figure 4 shows that a plume starts developing in the vertical mid-plane $(x=0)$ at $t=0.370$ due to the initial perturbation, and at $t=0.445$ the head of the plume begins descending. The plume has hit the bottom of the chamber at $t=0.483$. The variation of the central concentration, $n_{\text {mid }}=n\left(0, \frac{1}{2}\right)$, at the midpoint of the chamber (figure 5) shows that the plume becomes steady after a relatively short time. We have chosen the following method to determine whether a given solution is in steady state. The concentration fields from two different numerical solutions, usually 2000 time steps apart, are subtracted from each other at every grid point. If the maximum difference is less than $10^{-5}$, then we declare the solution to be steady.

Figure 6(a-c) shows the final steady-state cell concentration, total cell flux vector $\boldsymbol{J}$, and streamline contours respectively, whereas figure $7(a-c)$ shows the individual contributions of the advective cell flux, $\boldsymbol{u} n$, the diffusive cell flux, $\nabla n$, and the gyrotactic cell flux, $V_{c} \boldsymbol{p} n$, respectively, to the total cell flux vector $\boldsymbol{J}$.

To illustrate the dependence of the solution on the mesh, the properties of the above steady solution were compared on three different grids as shown in table 1, where the quantities given are

(i) the value of the concentration $n$ at the mid-point of the two-dimensional box;

(ii) the maximum value of the stream function $\psi$ together with its location;

(iii) the maximum value of the vorticity $\zeta$ on the rigid wall $(y=0)$, together with its location.

Since the solution is symmetric, the locations of $\psi_{\max }$ and $\psi_{\min }$ are symmetric about the $y$-axis and the same is true for the vorticity. The positions of the maximum and minimum values are obtained by a third-order polynomial approximation. It is seen from the table 1 that three-figure accuracy is achieved with $36 \times 36$ grid.

The above steady solution, classified as a one plume/two convection cell solution (the $2^{+}$solution of Lennie et al. 1988), of the bioconvection problem is not unique. There exist steady-state solutions with a single clockwise $\left(1^{+}\right)$convection cell and likewise one in an anti-clockwise sense $\left(1^{-}\right)$. Figure $8(a-c)$ shows one of these solutions, 
(a)
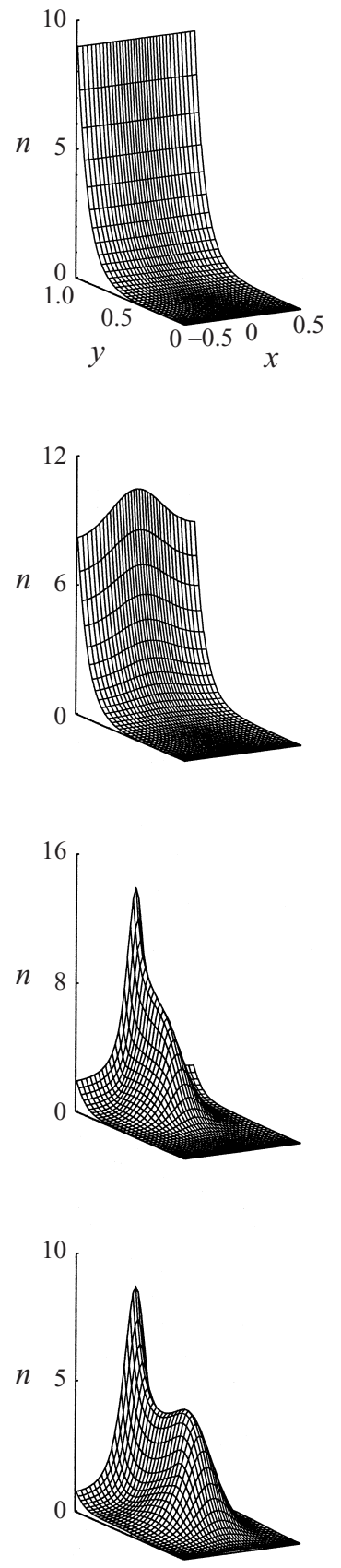

(b)
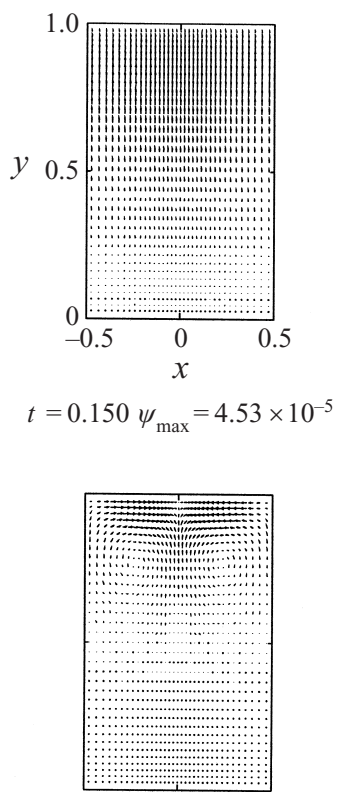

$t=0.370 \psi_{\max }=0.138$

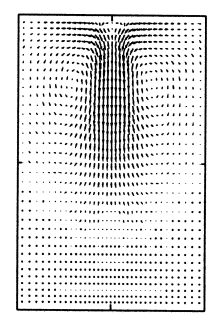

$t=0.445 \psi_{\text {max }}=2.150$

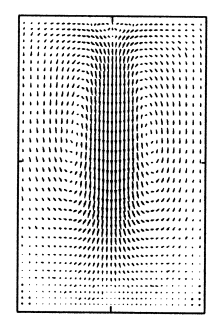

$t=0.483 \psi_{\text {max }}=2.143$ (c)
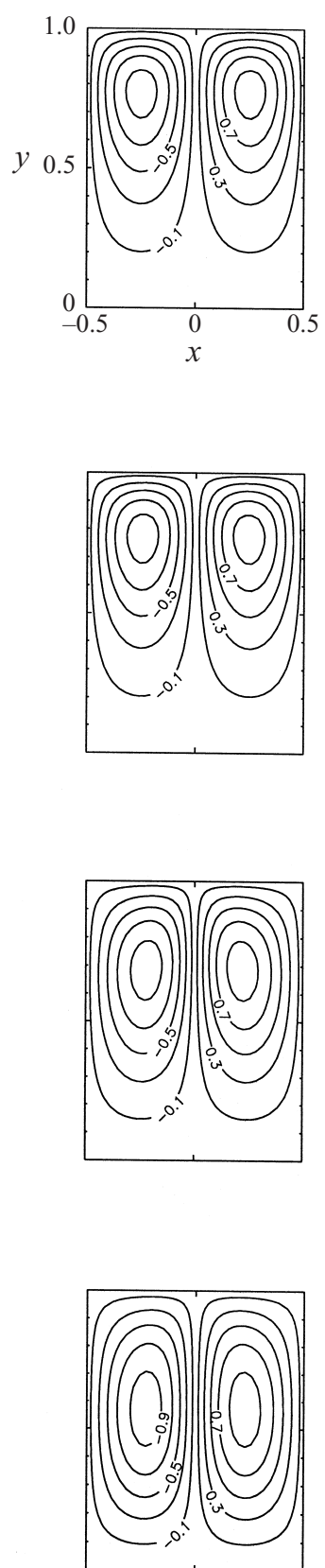

FIGURE 4. Gyrotactic plume formation for the suspension shown in figure 3 for aspect ratio $\lambda=1$ : $(a)$ concentration, $(b)$ cell fluxes, $(c)$ streamlines. The positive streamlines take values of $0.1,0.3,0.5,0.7,0.9$ of $\psi_{\max }$ with similar labelling for the negative streamlines. Here $\psi_{\max }=-\psi_{\min }$. 


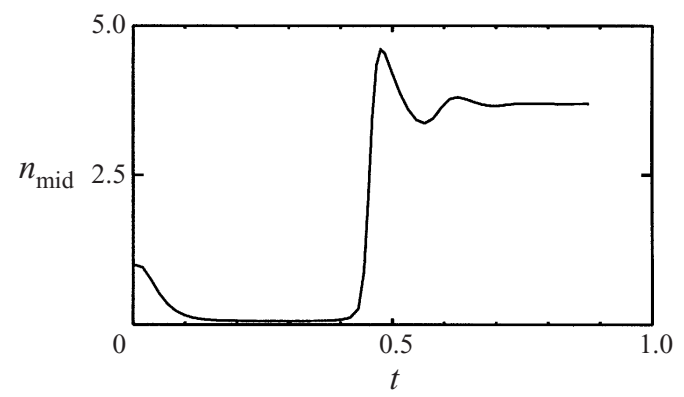

FIGURE 5. Variation of the central concentration with time for aspect ratio $\lambda=1$.

(a)

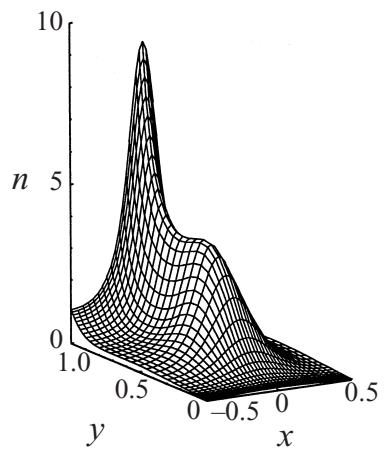

(b)

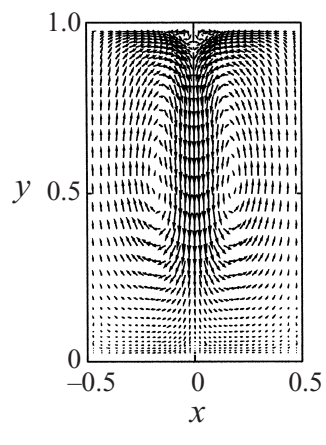

(c)

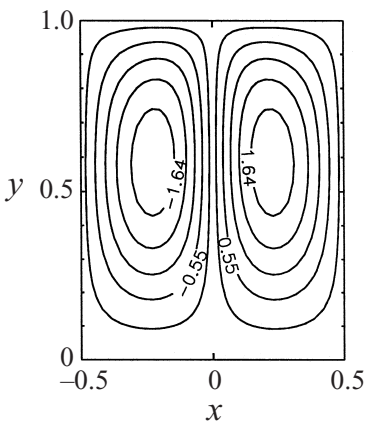

FIGURE 6. Steady-state plume $\left(2^{+}\right)$for aspect ratio $\lambda=1:(a)$ concentration, $(b)$ cell fluxes, (c) streamlines. The contour levels of the streamlines are equally spaced.

(a)

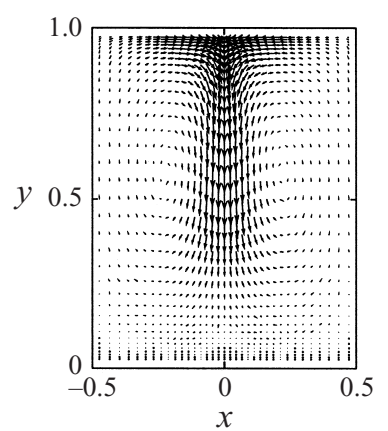

(b)

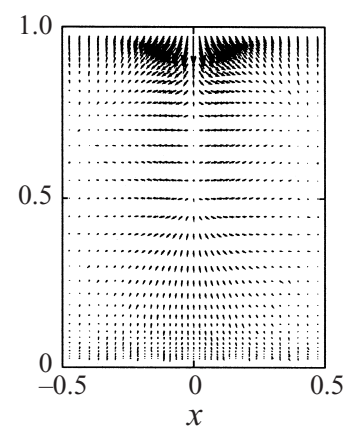

(c)

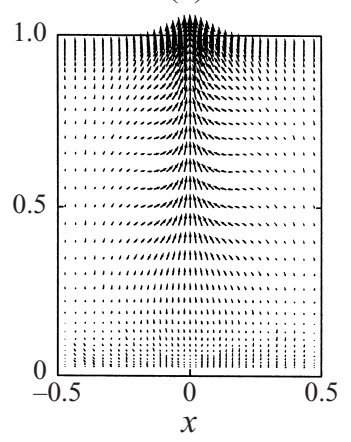

FIGURE 7. Steady-state cell fluxes for $\lambda=1$ due to $(a)$ advection, $(b)$ diffusion and $(c)$ gyrotaxis.

which arises from the initial cell distribution

$$
n=1+\epsilon \sin (m \pi x), \quad-\frac{1}{2} \leqslant x \leqslant \frac{1}{2},
$$

where $\epsilon=10^{-4}$ and $m=-1$. There also exists a two-plume (at $x= \pm \frac{1}{2}$ ) solution with two convection cells, which arises from the initial concentration distribution of the following form:

$$
n=1-\epsilon \cos (m \pi x), \quad-\frac{1}{2} \leqslant x \leqslant \frac{1}{2}
$$


(a)

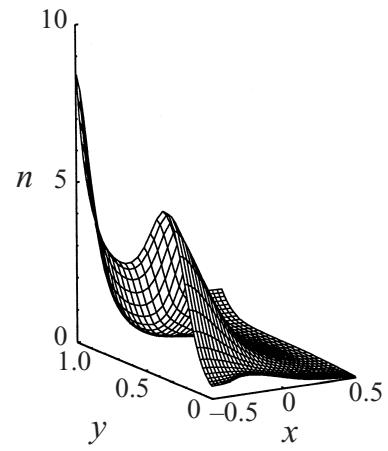

(b)

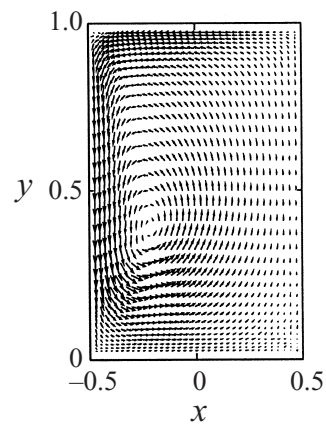

(c)

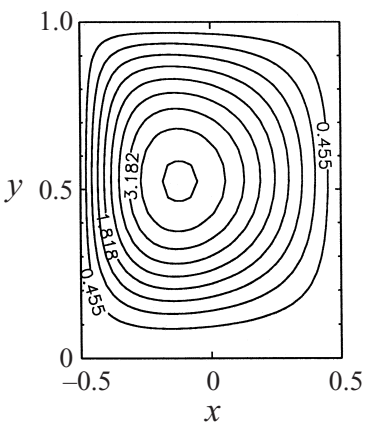

FIGURE 8. Steady-state plume $\left(1^{-}\right)$for aspect ratio $\lambda=1:(a)$ concentration, $(b)$ cell fluxes, (c) streamlines (equally spaced contour levels). This solution corresponds to initial cell distribution given by (23).

(a)

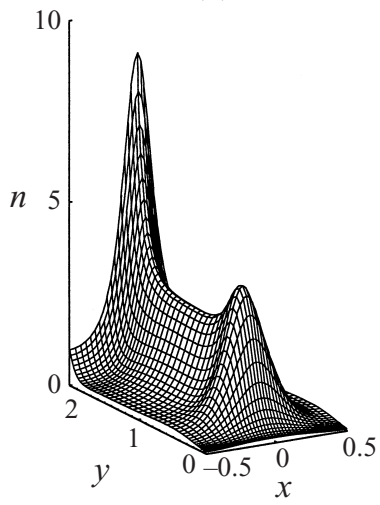

(b)

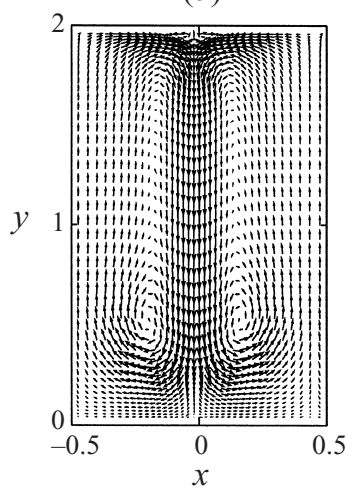

(c)



FIGURE 9. Gyrotactic solutions $\left(2^{+}\right)$of plume for aspect ratio $\lambda=2$ at $t=2.0$ : $(a)$ concentration, (b) cell fluxes, (c) streamlines (at equally spaced contour levels).

where $\epsilon=10^{-4}$ and $m=2$. This solution is termed the $2^{-}$solution by Lennie $e t$ al. (1988).

\subsection{Aspect ratio $\lambda=2$}

The concentration, cell fluxes and streamlines at $t=2$ for $\lambda=2$ are shown in the figure $9(a-c)$. It is seen from figure $9(b)$ that some of the cells are circulating at the bottom of the plume and that in the mid-region of the plume, the flow is almost independent of the vertical $y$-coordinate. If the solutions are independent of the $y$-coordinate, then from the equation of cell conservation, we find

$$
\frac{\mathrm{d}}{\mathrm{d} x} J_{x}=0 \quad \Rightarrow \quad J_{x} \equiv V_{c} p_{x} n-\frac{\mathrm{d} n}{\mathrm{~d} x}=C,
$$

where the constant $C=0$ since $\zeta=0$ and $\mathrm{d} n / \mathrm{d} x=0$ along the plume. Thus we have a balance between the horizontal diffusive flux and the horizontal gyrotactic flux. A plot of the horizontal gyrotactic cell flux, $\left.V_{c} p_{x} n\right|_{y=1}$, against the negative of the horizontal diffusive cell flux, $\partial n /\left.\partial x\right|_{y=1}$, verifies this conclusion (see figure $10 a$ ).

A plot of the central concentration, $n_{\text {mid }}=n(0,1)$, against time is shown in the 



FiguRE 10. (a) Comparison of the negative of the horizontal diffusive cell flux, $\mathrm{d} n / \mathrm{d} x(-)$ with the horizontal gyrotactic cell flux, $V_{c} p_{x} n\left(-*_{-*}\right)$ at $y=1$ for aspect ratio $\lambda=2$. (b) Variation of $n_{\text {mid }}=n(0,1)$ with time for aspect ratio $\lambda=2$.

(a) $t=1.11$

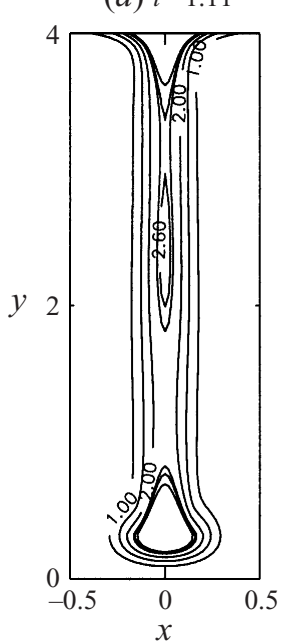

(b) $t=1.16$

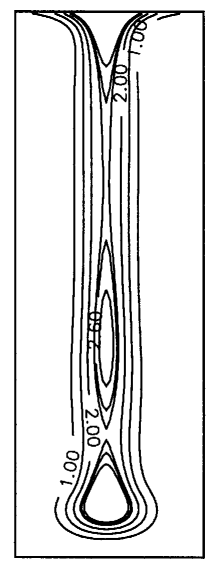

(c) $t=1.21$

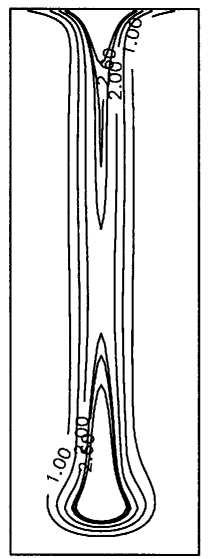

(d) $t=1.26$



FIGURE 11. One cycle of the oscillation in concentration (plotted as contour levels) for aspect ratio $\lambda=4$. The period is approximately 0.15 in non-dimensional units.

figure $10(b)$. The large value of $n(0,1)$ at around $t=0.25$ is the concentration at the head of the plume when it passes through the mid-point. The fluctuations in $n_{\text {mid }}$ are due to the occurrence of a small varicose 'blob'. The fluctuations take longer to disappear than when $\lambda=1$. The solution remains apparently in a steady state $\left(2^{+}\right.$solution) for a considerable amount of time. Ultimately, the perturbation due to rounding error grows and it shifts the plume to one of the stress-free sidewalls and a steady-state single convection cell (either $1^{+}$or $1^{-}$) forms with the fluid rotating in either the clockwise or anti-clockwise sense, similar to the solution shown in figure 8 .

\subsection{Aspect ratio $\lambda=4$}

In this case, the blob does not disappear; instead it convects along the plume periodically forming an intermediate state. Figure 11(a-d) shows one cycle of the oscillation. Figure 11 $(a)$ shows the blob just above the mid-height of the chamber and it is about to hit the bottom of the chamber in figure 11(b). Figure 11(c) shows the blob after it has hit the bottom of the chamber and figure 11(d) completes the cycle. 
(a) $t=2.61$

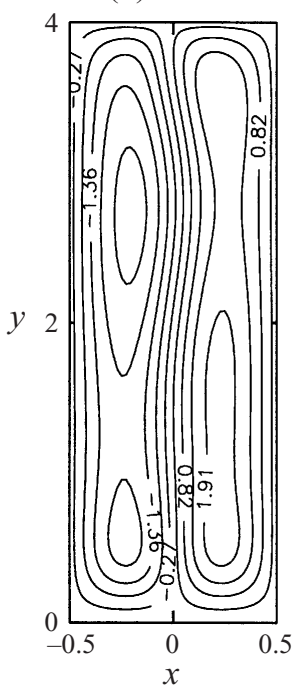

(b) $t=2.64$

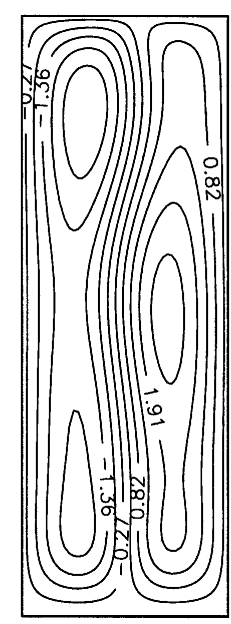

(c) $t=2.67$

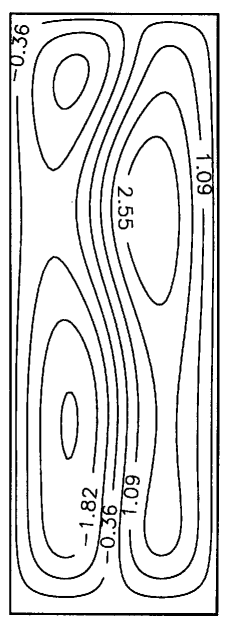

FIGURE 12. Growth of meandering instability: streamlines (equally spaced contour levels) for aspect ratio $\lambda=4$.

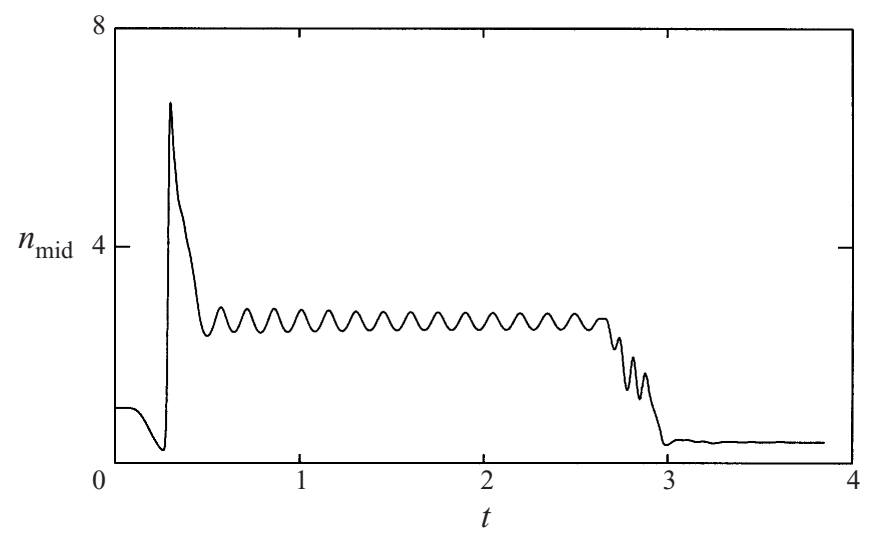

FIGURE 13. Variation of the central concentration, $n_{\text {mid }}=n(0,2)$, with time in a chamber of aspect ratio 4 . The drop in concentration at around $t=3$ indicates that the plume has shifted to one of the sidewalls via a meandering instability.

The wavelength of the varicose mode is the distance between two blobs. For aspect ratio 4 , this wavelength is a little less than 4 , since the two blobs are not located exactly at the top and the bottom of the chamber.

This periodic blob convection persists for a considerable amount of time, but ultimately a sideways 'meandering' instability develops. The development of this instability is shown in figure 12, where the streamlines are plotted at equal intervals of time. This meandering instability grows rapidly and the plume shifts from the centreline to one of the sidewalls. The wavelength of the meandering mode is also approximately 4 (see figure 12), the aspect ratio of the chamber.

The evolution of the central cell concentration, $n_{\text {mid }}=n(0,2)$, at the midpoint of the chamber from the initial uniform state to the final state of the plume is plotted 
(a) $t=1.50$

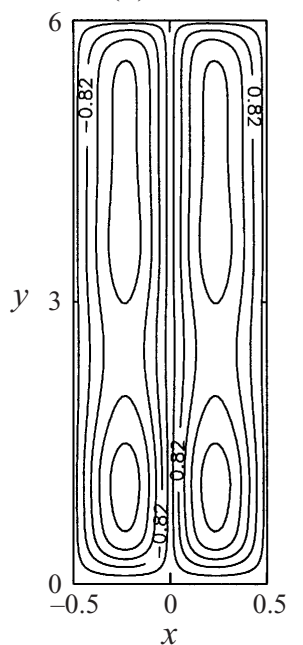

(b) $t=2.04$

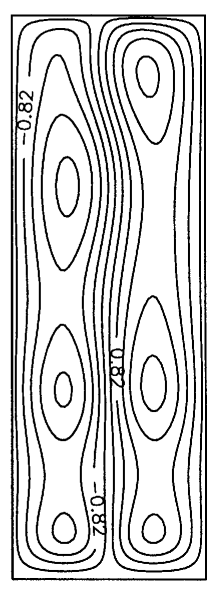

FIGURE 14. Snapshots of $(a)$ periodic blob convection and $(b)$ the growth of the meandering instability: streamlines for aspect ratio $\lambda=6$.

(a) $t=1.60$

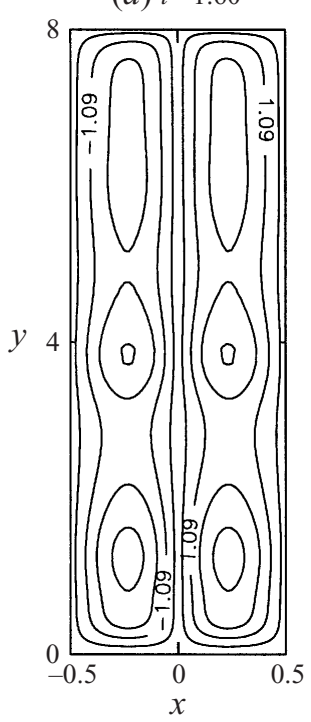

(b) $t=1.88$

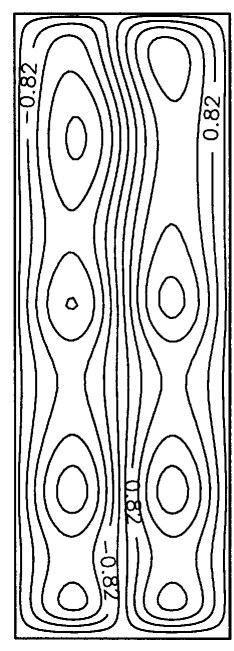

FIGURE 15. Snapshots of $(a)$ periodic blob convection and $(b)$ the growth of the meandering instability: streamlines for aspect ratio $\lambda=8$.

in figure 13. The fluctuation of $n_{\text {mid }}$ in $0.5 \leqslant t \leqslant 2.5$ corresponds to the periodic blob convection. This periodic behaviour is destroyed by the meandering instability (shown in figure 12) in the interval $2.5 \leqslant t \leqslant 3$ and ultimately the plume shifts to one of the sidewalls forming a single convection cell $\left(1^{ \pm}\right)$.

\subsection{Aspect ratios $\lambda=6$ and $\lambda=8$}

The streamlines for the aspect ratios 6 and 8 at two different times are shown in figure 14 and figure 15. Figure 14(a) shows periodic blob convection, where one blob 
(a) $t=1.5$

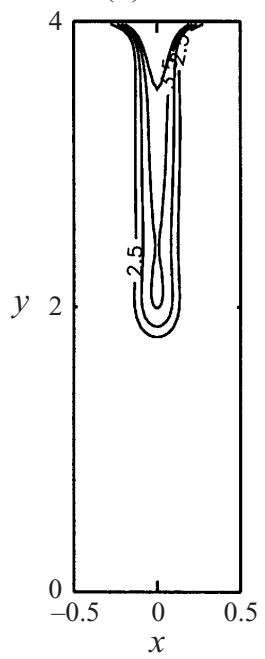

(b) $t=10.0$

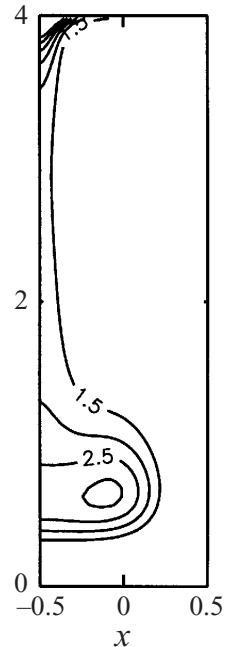

FIGURE 16. Concentration contours for $G=5 \times 10^{-3}(a)$ at an early stage and $(b)$ at final steady state for aspect ratio $\lambda=4$.

has hit the bottom of the chamber and another blob is approaching the mid-height. Figure 15(a) shows that after one blob has hit the bottom of the chamber, another is at the mid-height and another has formed at the top. Figure 14(a) is the intermediate state between solutions with one blob at $\lambda=4$ and two blobs at $\lambda=8$.

Figure 14(b) and figure 15(b) show the onset of the meandering instability for aspect ratios 6 and 8 respectively. These figures show that the meandering instability for $\lambda=8$ occurs after a shorter time than for $\lambda=6$, indicating that the growth rate of the meandering instability increases with an increase in the aspect ratio. The wavelength of the varicose mode is approximately 4 and that of the meandering mode is approximately 4 too based on the solution at $\lambda=8$.

\subsection{Parametric dependence}

Due to the large number of parameters, it is difficult to obtain a comprehensive picture across the whole parameter domain. Below we describe the results for a physically relevant sample of parameter values.

\subsubsection{Dependence on $G$}

The value of the gyrotaxis parameter, $G$, was varied in the range from $5 \times 10^{-3}$ to $1.5 \times 10^{-2}$, while the other parameters were kept constant:

$$
R=5 \times 10^{2}, \quad S_{c}=20, \quad V_{c}=10, \quad \lambda=4 .
$$

It was observed that for smaller values of the gyrotaxis number, the plumes do not extend down to the bottom of the chamber. The oscillations due to the 'blobs' begin to disappear after a relatively short time, but the meandering instability grows more rapidly. Ultimately this meandering instability destabilizes the plume and it shifts towards one of the stress-free sidewalls. Figure 16 shows the plume solution at two different times for $G=5 \times 10^{-3}$. The solution at $t=10$ is almost steady.

For intermediate values of $G$, the oscillations due to blobs persist longer, but still the meandering instability ultimately dominates over the varicose instability, 
(a) $G=0.01$

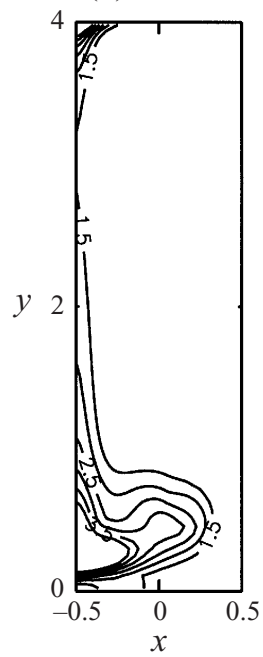

(b) $G=0.015$

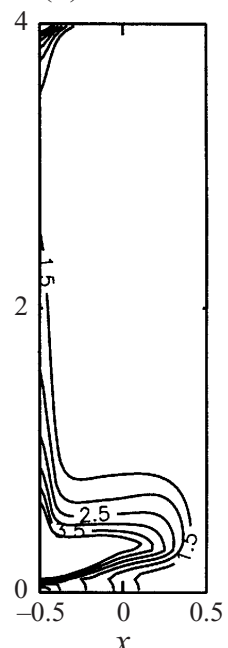

FIGURE 17. Almost steady-state concentration contours at $t=10$ for aspect ratio $\lambda=4$ when $G=0.01$ and 0.015 .

although it takes longer, and the plume shifts to one side. For large $G$, the blobs almost disappear, as most of the cells, after reaching the bottom of the chamber, are forced to remain there due to the strong circulation. Thus a large value of $G$ stabilizes the plume. The cells tumble for values of $G$ greater than approximately 0.012 . The weak effective horizontal gyrotactic swimming due to tumbling is a reason for the stabilization of the plume for large $G$. After a sufficiently long time, rounding errors in the computation gradually introduce perturbations, which begin to grow into the meandering instability. Ultimately this meandering instability breaks the plume down as in the previous cases. Figure 17 shows the steady-state concentration contours for $G=0.01$ and $G=0.015$. The cell concentration at the bottom of the chamber increases with an increase in the values of $G$.

The variation of the central concentration, $n_{\text {mid }}=n(0,2)$, at the midpoint of the chamber with time is shown in figure 18 for the three different values of $G$. It is seen that the time taken by the meandering instability to destabilize the plume increases with increase in the values of $G$. The oscillations due to blobs virtually disappear for large values of $G$. Sudden drops in the concentration values around $t=2,3$ and 9 in figure 18 indicate the shifting of the plume to one of the sidewalls. They show that the growth rate of the meandering instability decreases with increasing $G$ but the exact times also depend on the level of numerical rounding errors. This is discussed further in $\S 7$.

\subsubsection{Dependence on $V_{c}$}

Here we keep four of the parameters fixed at

$$
R=5 \times 10^{2}, \quad S_{c}=20, \quad G=10^{-2}, \quad \lambda=4
$$

and vary the cell swimming speed, $V_{c}$, in the range from 5 to 20 .

The properties of the solution with increase in $V_{c}$ are similar to those for the increase in $G$ except that the blob convection persists for larger values of $V_{c}$. Figure 19 shows the variation of the central concentration, $n_{\text {mid }}=n(0,2)$, at the midpoint of the 


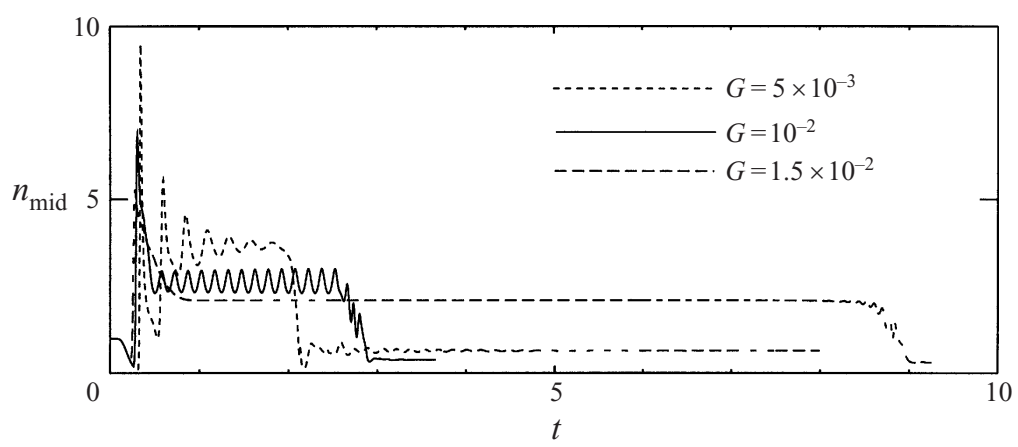

FIGURE 18. Effect of gyrotaxis number: variation of $n_{\text {mid }}=n(0,2)$ with time for three different values of $G$ at aspect ratio $\lambda=4$.

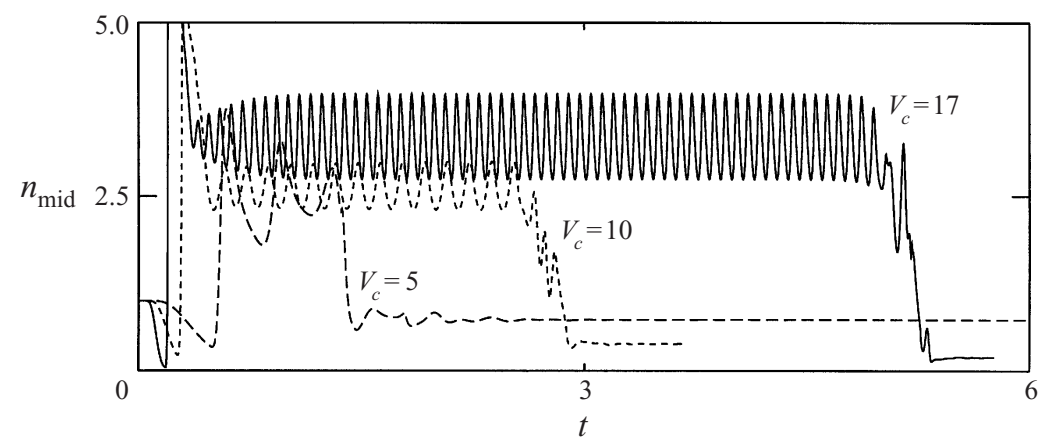

FIGURE 19. Effect of cell swimming speed: variation of $n_{\text {mid }}=n(0,2)$ with time for three different values of $V_{c}$ at aspect ratio $\lambda=4$.

chamber with time for three different values of $V_{c}$. The meandering instability sets in more quickly at smaller values of $V_{c}$.

\subsection{Remarks}

The $2^{+}$and $2^{-}$solutions in the steady state are equivalent and the same is true for the $1^{+}$and $1^{-}$solutions except the sense of fluid rotation. The solution with the central plume $\left(2^{+}\right)$has wavelength equal to the width of the chamber, whereas the solution with a plume on one of the sidewalls (either $1^{+}$or $1^{-}$) has a wavelength equal to twice the width of the chamber. Consequently, the critical Rayleigh numbers are different depending on whether the plume is initiated in the middle or at one of the sidewalls. The varicose mode can be simulated in a chamber of half the width of the original chamber, since the solution has symmetry about $x=0$. However, since the sidewalls are rigid and stress free with zero cell-flux through them, the meandering mode and its growth rate can only be studied on a plume at the middle of the chamber. Our initial conditions make the plume form in the middle of the chamber. Simulations without a perturbation to the initial uniform concentration usually lead to an initial disturbance in one of the corners at the top of the layer (due to accumulation of rounding errors) and result in one plume/single convection cell solutions $\left(1^{ \pm}\right)$.

When $G$ is small, the weak gyrotaxis is unable to pull enough cells from the top layer to extend the plume to the bottom of the chamber. However the $1^{ \pm}$plume is longer than the $2^{+}$plume. This is because the area of the region adjacent to the plume for $1^{ \pm}$solutions is twice that of the $2^{+}$solution so that the number of cells under the 


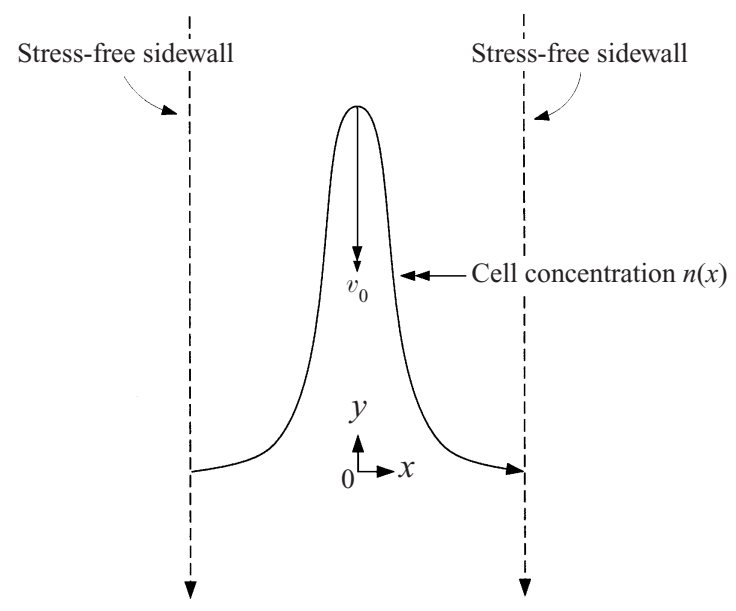

FIGURE 20. Geometry of an infinitely long plume located in $-\frac{1}{2} \leqslant x \leqslant \frac{1}{2}$ together with a schematic diagram of concentration profile. $v_{0}$ is the central downward speed of the plume.

influence of gyrotaxis is greater. Consequently, the effect of gyrotaxis is stronger for $1^{ \pm}$solutions than for the $2^{+}$solution.

\section{Linear stability of a gyrotactic plume}

We have seen two kinds of instabilities in a large aspect ratio chamber. In order to understand these instabilities better, a model of an infinitely long plume is derived and its linear stability is tested. The linear stability analysis is valid only when the cells do not tumble so that $G$ cannot be too large. The governing equations are (11)-(14) with the swimming direction given by

$$
\overline{\boldsymbol{p}}=\left(-G \zeta,\left(1-G^{2} \zeta^{2}\right)^{1 / 2}\right) .
$$

\subsection{The primary flow}

The basic flow configuration is sketched in figure 20. The central axis of the plume is located along the vertical $y$-axis and the flow is bounded by walls at $x= \pm \frac{1}{2}$. The walls are stress free and there is no flux of cells through them. Also the total nondimensional fluid flux, $Q_{f}$, and the total non-dimensional cell flux, $Q_{c}$, are prescribed. The values of both $Q_{f}$ and $Q_{c}$ are zero for the numerical experiments. We first seek the equilibrium steady fluid speed, $v(x)$, and the cell concentration, $n(x)$.

Application of the no-cell flux condition at the sidewalls to the equations of bioconvection, (11)-(14), gives

and

$$
\frac{\mathrm{d}^{3} v}{\mathrm{~d} x^{3}}=R \frac{\mathrm{d} n}{\mathrm{~d} x}
$$

$$
\frac{\mathrm{d} n}{\mathrm{~d} x}=-V_{c} G \frac{\mathrm{d} v}{\mathrm{~d} x} n .
$$

Equation (26) represents the balance between viscous stress and buoyancy and (27) represents the balance between the horizontal diffusive and the horizontal gyrotactic cell fluxes. Because of symmetry, the primary flow is solved for only in the region 
$0 \leqslant x \leqslant \frac{1}{2}$. The boundary conditions are

$$
\begin{gathered}
\frac{\mathrm{d} v}{\mathrm{~d} x}=0 \quad \text { at } x=0, \frac{1}{2}, \\
\int_{0}^{1 / 2} v(x) \mathrm{d} x=Q_{f} / 2,
\end{gathered}
$$

and

$$
\int_{0}^{1 / 2}\left[v(x)+V_{c}\left(1-G^{2} \zeta^{2}\right)^{1 / 2}\right] n(x) \mathrm{d} x=Q_{c} / 2 .
$$

Equation (27) can be integrated once giving

$$
n(x)=n_{0} \exp \left\{-V_{c} G\left(v+v_{0}\right)\right\},
$$

where $n_{0}=n(0)$ and $v_{0}=-v(0)$ are unknown constants and (26), on integrating twice and applying the boundary condition (28a), gives

$$
\left(\frac{\mathrm{d} v}{\mathrm{~d} x}\right)^{2}=\frac{2 R n_{0}}{T}\left\{1-\exp \left(-T\left(v+v_{0}\right)\right)-A T\left(v+v_{0}\right) \exp \left(-T v_{0}\right)\right\},
$$

where $T \equiv V_{c} G$ and $A$ is a constant of integration. Let $B$ be another constant defined so that $v\left(\frac{1}{2}\right)=B v_{0}$. Since $(\mathrm{d} v / \mathrm{d} x)\left(\frac{1}{2}\right)=0$, (30) implies that

$$
1-\exp \left(-T v_{0}(1+B)\right)-A T v_{0}(1+B) \exp \left(-T v_{0}\right)=0 .
$$

For a given value of $A$ and $B$, the product $T v_{0}$ (and hence $v_{0}$ ) is determined as a root of (31). On introducing $\omega=T\left(v+v_{0}\right)$, (30) can be integrated as

$$
\int_{0}^{\omega} \frac{\mathrm{d} \tilde{\omega}}{\sqrt{1-\exp (-\tilde{\omega})-A \tilde{\omega} \exp \left(-T v_{0}\right)}}=x\left(2 R n_{0} T\right)^{1 / 2} .
$$

When $x=\frac{1}{2}$, (32) reduces to

$$
\int_{0}^{T v_{0}(1+B)} \frac{\mathrm{d} \tilde{\omega}}{\sqrt{1-\exp (-\tilde{\omega})-A \tilde{\omega} \exp \left(-T v_{0}\right)}}=\left(2 R n_{0} T\right)^{1 / 2} / 2 .
$$

The integral in (33) has an integrable singularity at both end points and $n_{0}$ is determined from this equation. Once $n_{0}$ is determined, $\omega$ and hence $v(x)$ are found from (32), and then $n(x)$ from (29), for any $x \in\left[0, \frac{1}{2}\right]$. Equations (31), (28b) and (28c) are three simultaneous nonlinear equations in three unknowns $A, B$ and $T v_{0}$, which are solved numerically using iteration.

Figure 21 shows the basic flows for different values of $G$, the gyrotaxis number. The peak cell concentration is relatively low for large $G$ and vice versa. Figure 22 shows the effect of $V_{c}$ on the basic flows. The concentration gradient becomes steeper as the values of the cell swimming speed become larger but the peak cell concentration varies little.

\subsection{The linear stability problem}

We consider a small perturbation of amplitude $\epsilon(0<\epsilon \ll 1)$ to the equilibrium state, so that

$$
\psi=\psi(x)+\epsilon \psi^{*}(x, y, t), \quad n=n(x)+\epsilon n^{*}(x, y, t),
$$

and look for normal modes of the form

$$
\psi^{*}=\phi(x) \exp (\mathrm{i} \alpha(y-c t)) \quad \text { and } \quad n^{*}=\theta(x) \exp (\mathrm{i} \alpha(y-c t)) .
$$



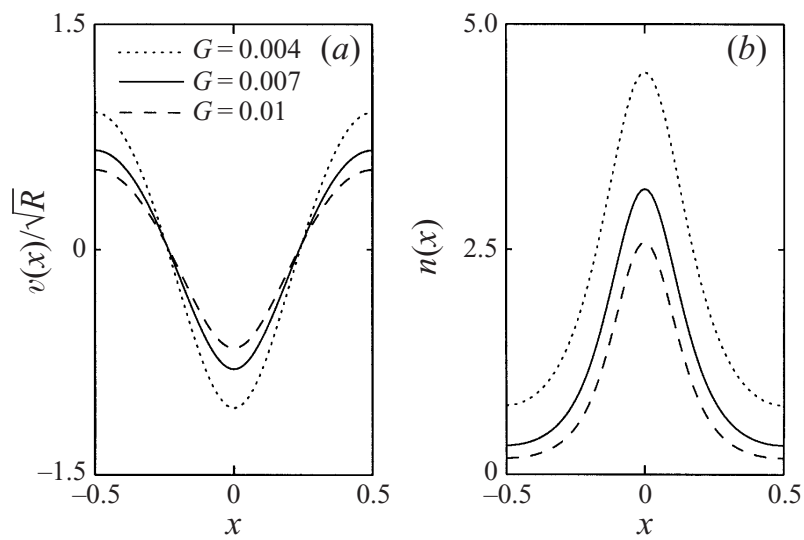

FiguRE 21. Basic velocity $(a)$ and concentration $(b)$ for an infinitely long plume for different values of $G$ in a flow between stress-free sidewalls. Here $R=500, V_{c}=10, Q_{f}=0$ and $Q_{c}=0$.
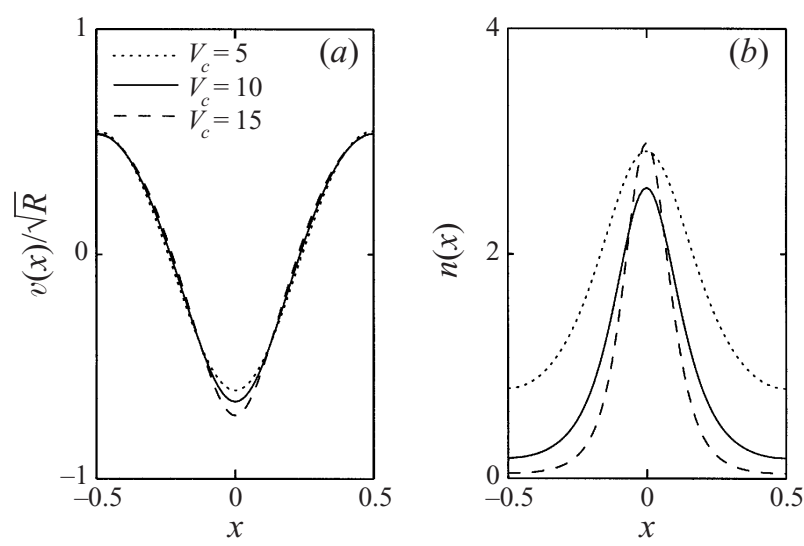

FiguRE 22. Basic velocity $(a)$ and concentration $(b)$ for an infinitely long plume for different values of $V_{c}$ in a flow between stress-free sidewalls. Here $R=500, G=0.01, Q_{f}=0$ and $Q_{c}=0$.

Substituting into the governing equations (11)-(13) and linearizing about the basic state gives at $O(\epsilon)$

$\mathrm{D}^{4} \phi=-R \mathrm{D} \theta+\left\{2 \alpha^{2}-\frac{\mathrm{i} \alpha c}{S_{c}}+\frac{\mathrm{i} \alpha v(x)}{S_{c}}\right\} \mathrm{D}^{2} \phi+\left\{\frac{\mathrm{i} \alpha^{3} c}{S_{c}}-\frac{\mathrm{i} \alpha}{S_{c}} \frac{\mathrm{d} \zeta}{\mathrm{d} x}-\frac{\mathrm{i} \alpha^{3} v(x)}{S_{c}}-\alpha^{4}\right\} \phi$

and

$$
\begin{aligned}
\mathrm{D}^{2} \theta= & -V_{c} G \zeta(x) \mathrm{D} \theta+\left\{\alpha^{2}-\mathrm{i} \alpha c+\mathrm{i} \alpha v(x)-V_{c} G \frac{\mathrm{d} \zeta}{\mathrm{d} x}+\mathrm{i} \alpha V_{c}\left(1-G^{2} \zeta^{2}\right)^{1 / 2}\right\} \theta \\
& +V_{c} G n(x) \mathrm{D}^{3} \phi+\left\{V_{c} G \frac{\mathrm{d} n}{\mathrm{~d} x}+\frac{\mathrm{i} \alpha V_{c} G^{2} \zeta(x) n(x)}{\left(1-G^{2} \zeta^{2}\right)^{1 / 2}}\right\} \mathrm{D}^{2} \phi-\alpha^{2} V_{c} G n(x) \mathrm{D} \phi \\
& +\left\{\mathrm{i} \alpha \frac{\mathrm{d} n}{\mathrm{~d} x}-\alpha^{2} V_{c} G \frac{\mathrm{d} n}{\mathrm{~d} x}-\frac{\mathrm{i} \alpha^{3} V_{c} G^{2} \zeta(x) n(x)}{\left(1-G^{2} \zeta^{2}\right)^{1 / 2}}\right\} \phi,
\end{aligned}
$$



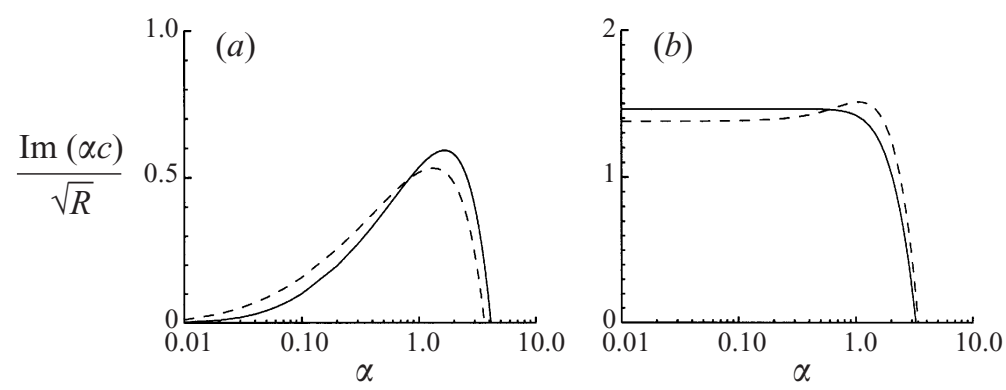

FIGURE 23. Growth rates as functions of the wavenumber, $\alpha$, of $(a)$ the varicose and $(b)$ the meandering instability modes of an infinitely long plume when $G=0.01(-)$ and $G=0.005$ $(---)$. Here $R=500, V_{c}=10, Q_{f}=0$ and $Q_{c}=0$.

where $\mathrm{D} \equiv \mathrm{d} / \mathrm{d} x ; v(x)$ and $\zeta(x)$ are the velocity and vorticity in the unperturbed equilibrium state. The boundary conditions are

$$
\phi=\mathrm{D}^{2} \phi=\mathrm{D} \theta=0 \quad \text { at } x= \pm \frac{1}{2} .
$$

This is an eigenvalue problem for $c$ as a function of the dimensionless parameters $R, S_{c}, V_{c}, G$ and $\alpha$, and the instability grows whenever $\operatorname{Im}(c)>0$.

\subsection{Numerical results}

Solutions to the full linear stability problem (35) to (37) are calculated with a fourthorder-accurate finite-difference scheme provided by Dr D. R. Moore (Cash \& Moore 1980). This scheme is used to compute (i) neutral curves for which $\operatorname{Im}(c)=0$, and (ii) the growth rate as a function of $\alpha$ for a given values of the parameters. Initially, values of $R, G, V_{c}, S_{c}$ and $\alpha$ are supplied, and the values of $c, \phi$ and $\theta$ are estimated either from the asymptotic results, or from the previous numerical results, or by imposing a sinusoidal variation in $\phi(x)$ and $\theta(x)$. Once a solution is obtained, this solution can be used as an initial guess for the neighbouring parameter values. The dependence of the numerical solutions on the number of grid points was tested for different parameter values. The orders of magnitude of the growth rates at long wavelengths agree with those predicted by the asymptotic analysis (see the Appendix). It is clear from the nature of (35) and (36) that if $\phi(x)$ is an odd function in $x$, then $\theta(x)$ must be an even function. This is the varicose mode. For the meandering mode, $\phi(x)$ is even and $\theta(x)$ is odd in $x$.

\subsubsection{Effect of the gyrotaxis number}

The growth rate curves associated with two different values of $G$ are plotted in figure 23. Figure $23(a)$ shows that, for the varicose mode, the plume with the larger gyrotaxis number is more unstable at large wavenumbers and vice versa. The dependence of the growth rate for the meandering mode on $G$ is opposite to that of the varicose mode (see figure $23 b)$. Plumes are always unstable $(\operatorname{Im}(c)>0)$ at small wavenumbers (long wavelengths), but unconditionally stable $(\operatorname{Im}(c)<0)$ at sufficiently large wavenumbers (small wavelengths). The peaks in the growth rate curves (figure 23) correspond to the most unstable wavenumbers. The value of $\operatorname{Re}(c)$ is non-zero for both the varicose and the meandering modes.

Figure 24 shows the stability diagram in the $(G, \alpha)$-plane. It is seen that the plane can be divided into five regions. In region I, the plume is unstable to both the varicose and the meandering modes, but the growth rate of the meandering mode 




FIGURE 24. Stability diagram for an infinitely long plume in the $(G, \alpha)$-plane. Here $R=500, V_{c}=10$, $Q_{f}=0$ and $Q_{c}=0$. The growth rate of the varicose mode $\left(V_{g}\right)$ is zero on the long-dashed line and that of the meandering mode $\left(M_{g}\right)$ is zero on the short-dashed line, and $V_{g}=M_{g}$ on the solid line.

$\left(M_{g}\right)$ is greater than that of the varicose mode $\left(V_{g}\right)$. Thus the plume becomes unstable via a meandering instability at small wavenumbers (region I). For large values of $G$ and moderate values of the wavenumbers (regions II and IV), the plume becomes unstable via a varicose instability. On the other hand, for small values of $G$ and moderate values of the wavenumbers (region III), the plume becomes unstable via a meandering instability. The plume is unconditionally stable at sufficiently large wavenumbers (region V).

\subsubsection{Effect of the cell swimming speed}

The behaviour of the growth rates and the associated linear stability diagram when $V_{c}$ is varied are similar to those when the gyrotaxis number is varied. Plumes with large (small) values of $V_{c}$ are more unstable at large (small) wavenumbers to the varicose mode and the opposite is true for the meandering mode. The plumes become unstable via the meandering mode at small wavenumbers for all values of $V_{c}$ and at moderate wavenumbers for smaller values of $V_{c}$. On the other hand for moderate values of the wavenumbers and larger values of $V_{c}$, the plume becomes unstable to the varicose mode. The plume is unconditionally stable at sufficiently large wavenumbers.

\subsubsection{Instability mechanism}

We have plotted the instability growth rate curves for the infinitely long plume in figure 23 for the parameter values (shown in the caption) that were used in the numerical experiments to examine the effect of the aspect ratio on the plume's structure and stability. For the varicose mode, the critical wavelength is approximately 1.5 and the most unstable wavelength is approximately 4 . For the meandering mode, the critical wavelength is approximately 2 and the most unstable wavelength is approximately 17, but all the wavelengths in the range from 6 to $\infty$ have growth rates nearly equal to the growth rate of the most unstable wavelength. Also, the meandering mode dominates the varicose mode for wavelengths in the range from 2.4 to $\infty$. The instability associated with the varicose mode leads to blob formation, whereas a meandering mode leads to the formation of a sinuous plume. The contours of the perturbed concentration are plotted for the varicose mode over a wavelength of 4 in figure 25(a) and for the meandering mode over a wavelength of 6 in figure $25(b)$.

The mechanism for the varicose instability is shown schematically in figure $26(a)$. The bulging region of the plume corresponds to positively perturbed concentration 
(a)

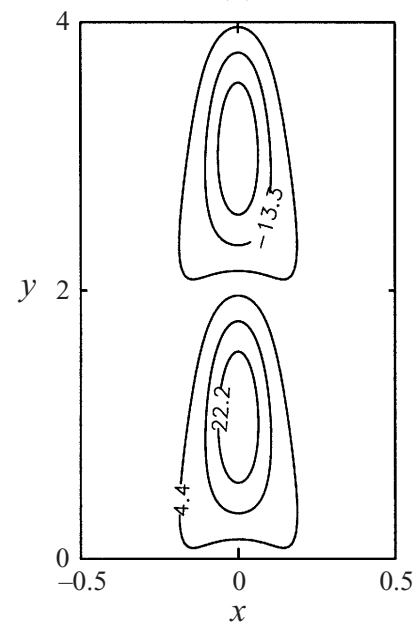

(b)

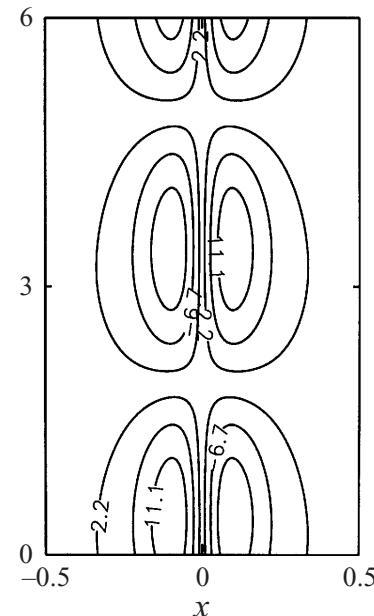

FIGURE 25. Contours of the perturbed concentration $n^{\prime}(x, y)$ for $(a)$ the varicose and $(b)$ meandering instability modes for an infinitely long plume. Here $R=500, G=0.01, V_{c}=10, Q_{f}=0$ and $Q_{c}=0$.

and the narrow region corresponds to negatively perturbed concentration. The region of higher cell concentration sinks downwards because it is more negatively buoyant, and introduces circulations as shown in figure 26(a). These circulations attract more cells due to gyrotaxis from nearby and reinforce the bulging region. The region of lower concentration induces circulations in the opposite sense and hence the concentration in that region depletes further.

The mechanism for the meandering instability is shown schematically in figure $26(b)$. The region where the plume bends radially outwards sinks because it is negatively buoyant and causes circulations as shown in figure $26(b)$. These circulations lead to the accumulation of more cells in the regions indicated by downward pointing arrows and depletion of cells on the opposite side. Thus gyrotaxis also enhances the formation of this mode.

\section{Conclusions}

It is instructive to compare the instability mechanisms and wavelengths for the infinitely long plume to those of the plume in a chamber of finite depth. Numerical experiments in a chamber of finite depth, with a rigid and no-slip bottom wall and a stress-free top wall were performed in $\S 5$. We observed the occurrence of a varicose mode for aspect ratio 2 during the evolution of the plume, but this ultimately disappears. This is presumably due to the constraints imposed by the boundary conditions at the top and bottom boundaries. At higher aspect ratios, the blob persist for longer and are convected along the plume periodically. Ultimately, however, the plume becomes unstable to a meandering instability. There is one blob at aspect ratio 4 , but this increases to two for aspect ratio 8, which indicates that the varicose mode has a finite most unstable wavelength. The growth rate curve for the varicose mode (see figure 23) on an infinite plume predicts this value to be approximately 4 , which is consistent with the numerical evidence for a finite-depth plume.

We observed that the plume at aspect ratio 1 was stable, but at aspect ratio 2 it was unstable to the meandering mode when given a small perturbation, although the 

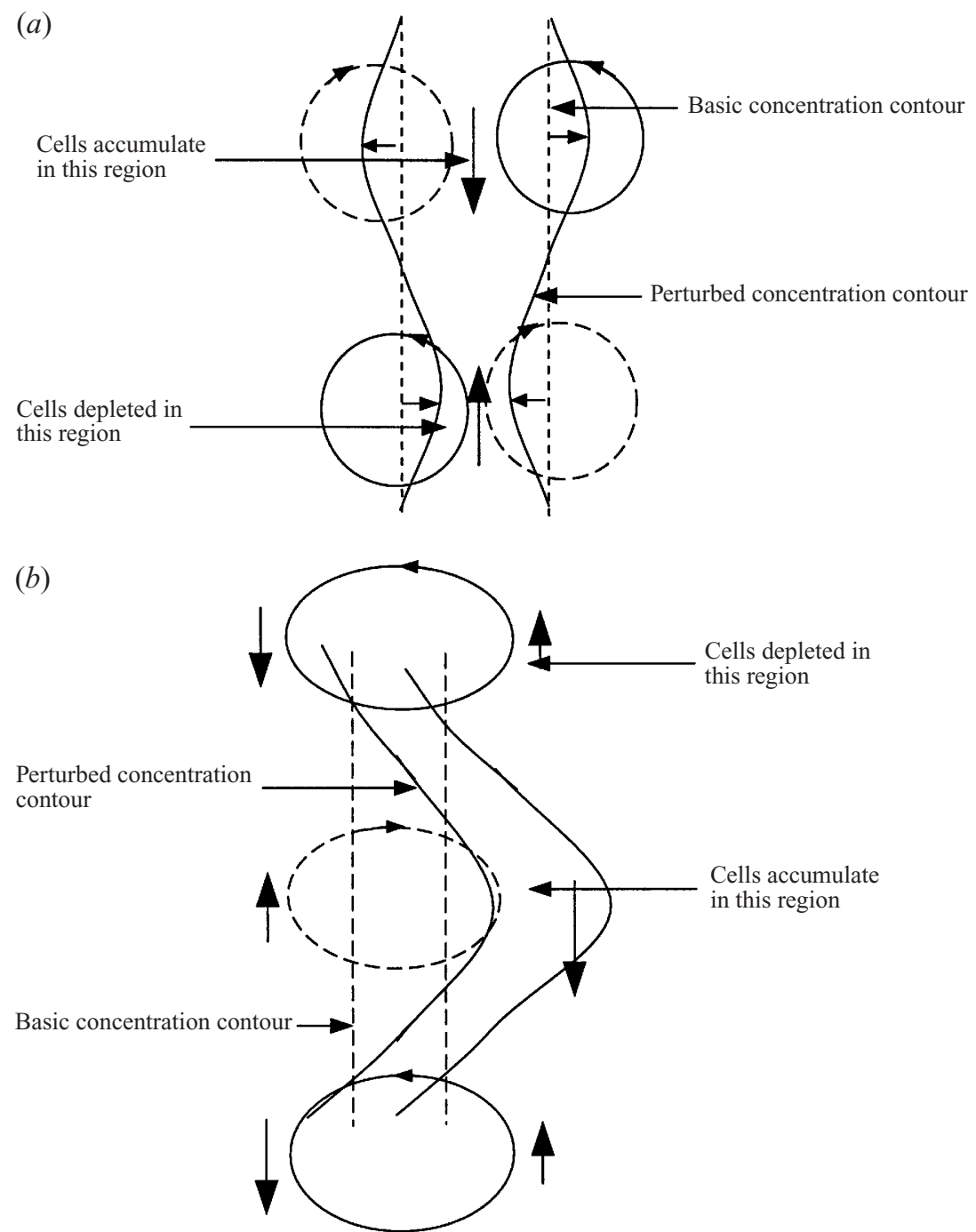

FIGURE 26. The mechanism for the $(a)$ varicose and $(b)$ meandering instability. The regions marked with downward (upward) pointing arrows are more (less) negatively buoyant and cause circulation in the sense shown in the figure.

growth rate of this mode was small. This suggests that the critical wavelength is less than 2 due to the non-periodicity of the top and bottom boundaries. The estimate for the most unstable wavelength for the meandering mode, based on numerical experiments at aspect ratios 4, 6 and 8, is approximately 4 . Both of these values are lower than those predicted by the infinite-depth linear stability theory. This underestimate is likely to be due to several reasons. First the presence of the large cell concentration at the top of the plume amplifies the growth rate to such an extent that the top of the plume shifts to one of the sidewalls before the meandering disturbance reaches the bottom of the chamber. Also, due to the strong circulation and the no-slip bottom wall, the lowest part of the plume is less susceptible to horizontal movement. Thirdly, in the numerical experiments, the meandering instability develops in the presence of the varicose instability and the vertical wavelength of the meandering mode appears to be constrained approximately between two varicose blobs. 


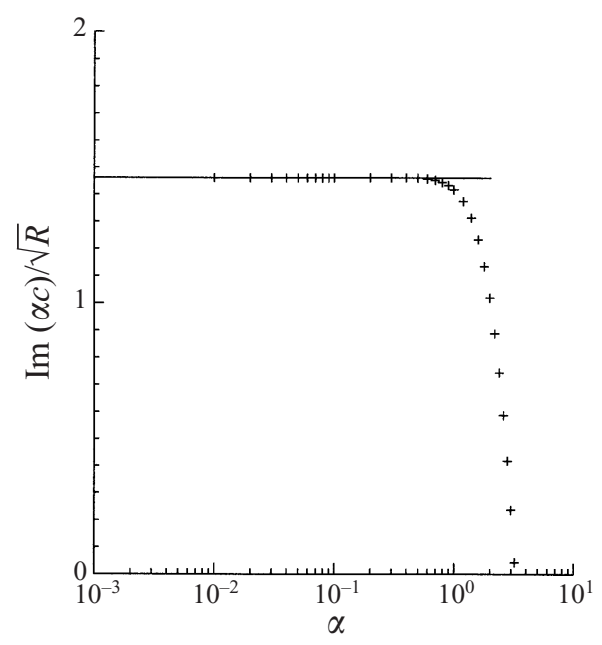

FiguRE 27. Comparison between full numerical solutions $(+)$ and the asymptotic solutions (solid lines) of the linear stability problem for the infinitely long plume when $R=500$, $G=0.01, V_{c}=10, Q_{f}=0$ and $Q_{c}=0$.

There is also good agreement between the linear stability analysis for an infinite plume and numerical experiments in a chamber of aspect ratio 4 as various parameters are varied. For values of $G$ in the range from 0.005 to 0.015 , following an initial varicose instability, the plume reaches the critical meandering instability stage rapidly when $G=0.005$. When $G$ is increased to 0.01 , the varicose instability persists longer, i.e. the growth rate of the meandering mode decreased as $G$ is increased from small to intermediate values. When $G=0.015$, the blobs almost disappear, i.e. the growth rate of the varicose mode again decreases. Linear stability analysis also suggests that the meandering mode is dominant at moderate wavelengths for smaller values of $G$ and the growth rate of the varicose (meandering) mode increases (decreases) as $G$ is increased from small to intermediate values (see figures 23 and 24). The linear stability analysis is not applicable for $G>0.012$ since the cells tumble for $G>0.012$. We also performed numerical experiments in a chamber of aspect ratio 4 for values of $V_{c}$ in the range from 5 to 15 . The time taken to reach the critical meandering stage increases with an increase in the values of $V_{c}$. Thus the growth rate of the varicose (meandering) mode increases (decreases) with an increase in the value of $V_{c}$, which is also predicted by the linear stability analysis.

Linear stability analysis predicts that the meandering instability is the most unstable mode for a wavelength greater than approximately 2.4 (see figure 23 ) when $G=$ $0.01, R=500$ and $V_{c}=10$. In our numerical experiments, we always observed the varicose instability appearing first since the initial condition (18) strongly favours the varicose mode over the meandering mode. This is because the initial condition and the equations are symmetric under reflection in $x$ so that the solution will remain symmetric until numerical rounding errors become significant and trigger the meandering instability. Thus the varicose instability is forced to appear at the beginning, but ultimately the meandering instability is dominant.

Time-dependent varicose instabilities or 'blobs' on plumes in bioconvection are studied here. This phenomenon is not new to convection problems. Lennie et al. (1988) found similar structures in the thermal convection of a Boussinesq fluid with an infinite Prandtl number confined between rigid boundaries. The time dependence 
is related to the formation of hot or cold blobs in the unstable thermal boundary layers. The varicose instability described in this paper is similar to the bamboo waves (Bai, Chen \& Joseph 1992) generated in a vertical pipe upflow when more viscous, less dense oil in the core flow is encircled by less viscous, more dense water. Lister (1987) investigated the stability of the two-dimensional flow due to the steady fall of a narrow, laminar plume: a dense fluid falling through a lighter fluid. The plume is found to be unstable to both varicose and meandering modes. The fundamental cause of the instability in the last two references is the contrast in viscosity between the two fluids. On the other hand, Smith (1989) analysed the stability of a vertical column of one fluid on the axis of a cylinder containing another, both acted upon by a uniform pressure gradient. The densities of the two fluids were different but their viscosities were the same. The mechanism for this instability is based on the interaction between the shear stress and the pressure. The present system is a single fluid with constant viscosity, unlike the two-fluid systems of Bai et al. (1992), Lister (1987) and Smith (1989), where a clear boundary exists between the two fluids. The role of gyrotaxis can be drawn out from the following argument. First, it helps in the formation of a concentrated line plume due to gyrotactic focusing. Secondly, suppose we switch off the gyrotaxis and cell diffusion in an existing concentrated plume. The linear stability equation for the cell conservation equation becomes

$$
c \theta=\phi \frac{\mathrm{d} n}{\mathrm{~d} x}+\left(v(x)+V_{c}\right) \theta .
$$

The boundary condition on $\theta$ is

$$
\theta=0 \quad \text { at } x=0 \text { for the meandering mode. }
$$

We specify

$$
\theta=1 \quad \text { at } x=0 \text { for the varicose mode }
$$

to fix the amplitude of the perturbation.

Since $\mathrm{d} n / \mathrm{d} x=0$ at $x=0,(38)$ is satisfied at $x=0$ for any value of $c$ for the meandering mode, whereas for the varicose mode $c$ is real. Thus, the meandering instability is possible without gyrotaxis but gyrotaxis enhances the meandering mode by the mechanism described in $\S 6.3 .3$. On the other hand, gyrotaxis is essential for the varicose instability.

Some aspects of the cell suspension that require further study include the following:

(i) Cell geometry For simplicity, we have considered spherical cells only, whereas a typical algal cell closely resembles a spheroid. Also the effects of the flagella were ignored.

(ii) Randomness in cell swimming trajectories In this paper, the randomness in the cells' swimming trajectories is modelled by a constant isotropic diffusion coefficient, $D$, (see (5)) and the mean swimming direction, $\overline{\boldsymbol{p}}$, is given deterministically by (15). Although this has the benefit of simplicity and is a good first model for gyrotaxis, it is ad hoc. Pedley \& Kessler (1990) have proposed a more rational model in which the mean swimming direction and the diffusion are derived from the probability density function for the orientation of the cells, which itself is governed by a Fokker-Planck equation. Bees, Hill \& Pedley (1998) and Bees \& Hill $(1998,1999)$ have obtained analytical results for a continuum model of bioconvection which incorporates the Fokker-Planck equation. The new model remains a challenge for future numerical solution. 
S.G. gratefully acknowledges funding from an Overseas Research Studentship and from the Tetley and Lupton Foundation of the University of Leeds.

\section{Appendix. Solutions valid at long wavelengths}

Due to the nonlinear coefficients in (35) and (36), it is difficult to solve these equations analytically, but in the limit of long wavelengths, i.e. $\alpha \rightarrow 0$, the order of the linear stability equations can be reduced and the growth rate can be estimated analytically. Since the order of the growth rate of the meandering mode $(\theta(x)$ odd) is higher than that of the varicose mode $(\theta(x)$ even) at long wavelengths, only the solutions for the meandering mode are presented.

The boundary conditions are

$$
\begin{array}{ll}
\mathrm{D} \phi=\mathrm{D}^{3} \phi=\theta=0 & \text { at } \quad x=0, \\
\phi=\mathrm{D}^{2} \phi=\mathrm{D} \theta=0 & \text { at } \quad x=\frac{1}{2}
\end{array}
$$

and

$$
\mathrm{D}^{2} \phi=1 \quad \text { at } \quad x=0 .
$$

The boundary condition (A $1 c$ ) is used to fix the amplitude of the perturbation.

Let

$$
\begin{aligned}
\phi & =\phi_{0}+\alpha \phi_{1}+\alpha^{2} \phi_{2}+\ldots, \\
\theta & =\theta_{0}+\alpha \theta_{1}+\alpha^{2} \theta_{2}+\ldots
\end{aligned}
$$

and

$$
c=\alpha^{-1} c_{-1}+c_{0}+\alpha c_{1}+\alpha^{2} c_{2}+\ldots
$$

Substituting into (35), (36) and into the boundary conditions (A 1a)-(A 1c), we find at leading order, $O(1)$, that

$$
\mathrm{D}^{4} \phi_{0}+R \mathrm{D} \theta_{0}=-\frac{\mathrm{i} c_{-1}}{S_{c}} \mathrm{D}^{2} \phi_{0}
$$

and

$$
\mathrm{D}^{2} \theta_{0}+V_{c} G \mathrm{D}\left(\zeta(x) \theta_{0}\right)-V_{c} G \mathrm{D}\left(n(x) \mathrm{D}^{2} \phi_{0}\right)=-\mathrm{i} c_{-1} \theta_{0},
$$

with boundary conditions

and

$$
\begin{array}{ll}
\mathrm{D} \phi_{0}=\mathrm{D}^{3} \phi_{0}=\theta_{0}=0 & \text { at } x=0, \\
\phi_{0}=\mathrm{D}^{2} \phi_{0}=\mathrm{D} \theta_{0}=0 & \text { at } x=\frac{1}{2}
\end{array}
$$

$$
\mathrm{D}^{2} \phi_{0}=1 \quad \text { at } x=0 .
$$

Now suppose that $\varpi=\int_{1 / 2}^{x} \theta_{0} \mathrm{~d} x$. Integrating (A 2) and (A 3) using the boundary conditions, we get

and

$$
\mathrm{D}^{2} \phi_{0}+R \varpi=-\frac{\mathrm{i} c_{-1}}{S_{c}} \phi_{0}
$$

$$
\mathrm{D}^{2} \varpi+V_{c} G \zeta(x) \mathrm{D} \varpi-V_{c} G n(x) \mathrm{D}^{2} \phi_{0}=-\mathrm{i} c_{-1} \varpi,
$$

subject to the boundary conditions

$$
\begin{aligned}
& \mathrm{D} \varpi=\mathrm{D} \phi_{0}=0 \quad \text { at } \quad x=0, \\
& \phi_{0}=\varpi=0 \quad \text { at } \quad x=\frac{1}{2}
\end{aligned}
$$


and

$$
R \varpi+\frac{\mathrm{i} c_{-1}}{S_{c}} \phi_{0}=-1 \quad \text { at } x=0 .
$$

Multiplying (A 5) by $\mathrm{D}^{2} \bar{\phi}_{0}$, where $\bar{\phi}_{0}$ denotes the complex conjugate of $\phi_{0}$, and integrating from $x=0$ to $x=\frac{1}{2}$, we get

$$
\int_{0}^{1 / 2}\left|\mathrm{D}^{2} \phi_{0}\right|^{2} \mathrm{~d} x=\frac{\mathrm{i} c_{-1}}{S_{c}} \int_{0}^{1 / 2}\left|\mathrm{D} \phi_{0}\right|^{2} \mathrm{~d} x-R \int_{0}^{1 / 2} \varpi \mathrm{D}^{2} \bar{\phi}_{0} \mathrm{~d} x
$$

Integrating by parts and using the mean value theorem, we get the following identities:

$$
\begin{aligned}
\int_{0}^{1 / 2} \varpi \mathrm{D}^{2} \bar{\varpi} \mathrm{d} x & =-\int_{0}^{1 / 2}|\mathrm{D} \varpi|^{2} \mathrm{~d} x . \\
\int_{0}^{1 / 2} \zeta(x) \varpi \mathrm{D} \bar{\varpi} \mathrm{d} x=-\int_{0}^{1 / 2} v(x)|\mathrm{D} \varpi|^{2} \mathrm{~d} x+v(\xi) \int_{0}^{1 / 2}|\mathrm{D} \varpi|^{2} \mathrm{~d} x & \text { for } \quad 0 \leqslant \xi \leqslant \frac{1}{2}, \\
\int_{0}^{1 / 2} n(x) \varpi \mathrm{D}^{2} \bar{\phi}_{0} \mathrm{~d} x=n(\tau) \int_{0}^{1 / 2} \varpi \mathrm{D}^{2} \bar{\phi}_{0} \mathrm{~d} x & \text { for } \quad 0 \leqslant \tau \leqslant \frac{1}{2} .
\end{aligned}
$$

Taking the complex conjugate of (A 6), multiplying by $\varpi$ and integrating from $x=0$ to $x=\frac{1}{2}$ (using the above identities), we get

$$
\int_{0}^{1 / 2}\left[1-V_{c} G(v(\xi)-v(x))\right]|\mathrm{D} \varpi|^{2} \mathrm{~d} x-\mathrm{i} c_{-1} \int_{0}^{1 / 2}|\varpi|^{2} \mathrm{~d} x+V_{c} G n(\tau) \int_{0}^{1 / 2} \varpi \mathrm{D}^{2} \bar{\phi}_{0} \mathrm{~d} x=0 .
$$

Elimination of

$$
\int_{0}^{1 / 2} \varpi \mathrm{D}^{2} \bar{\phi}_{0} \mathrm{~d} x
$$

from (A 8) and (A 9) shows that $c_{-1}$ is purely imaginary at leading order and thus determines the stability of plume. Since $c_{-1}$ is purely imaginary, the eigenfunctions are real and at leading order only a fifth-order system given by (A 5) to (A 6) needs to be solved to determine the eigenvalues and eigenfunctions. Since $c_{-1}$ is purely imaginary, the modes are non-oscillatory at long wavelengths. Figure 27 shows that there is good agreement between the asymptotic and numerical solutions.

\section{REFERENCES}

BAI, R., Chen, K. \& Joseph, D. D. 1992 Lubricated pipelining: stability of core annular flow. Part 5. Experiments and comparison with theory. J. Fluid Mech. 240, 97-132.

BatcheloR, G. K. 1970 The stress system in a suspension of force-free particles. J. Fluid Mech. 41, $545-570$

Bees, M. A. \& Hill, N. A. 1997 Wavelengths of bioconvection patterns. J. Expl Biol. 200, 1515-1526.

Bees, M. A. \& Hill, N. A. 1998 Linear bioconvection in a suspension of randomly swimming, gyrotactic micro-organisms. Phys. Fluids A 10, 1864-1881.

BeEs, M. A. \& Hill, N. A. 1999 Non-linear bioconvection in a deep suspension of gyrotactic swimming micro-organisms. J. Math. Biol. 38, 135-168.

Bees, M. A., Hill, N. A. \& Pedley, T. J. 1998 Analytical approximations for the orientation distributions of small dipolar particles in steady shear flows. J. Math. Biol. 36, 269-298.

CASH, J. R. \& MoORE, D. R. 1980 A high order method for the numerical solution of two point boundary value problems. BIT 20, 44-52.

Childress, S., Levandowsky, M. \& Spiegel, E. A. 1975 Pattern formation in a suspension of swimming micro-organisms: equations and stability theory modes. J. Fluid Mech. 69, 595-613.

GHORAI, S. 1997 Bioconvection and plumes. PhD thesis, University of Leeds. 
Harashima, A., Watanabe, M. \& Fujishiro, I. 1988 Evolution of bioconvection patterns in a culture of motile flagellates. Phys. Fluids 31, 764-775.

Hill, N. A. \& HäDER, D. P. 1997 A biased random random walk model for the trajectories of swimming micro-organisms. J. Theor. Biol. 186, 503-526.

Hill, N. A., Pedley, T. J. \& Kessler, J. O. 1989 Growth of bioconvection patterns in a suspension of gyrotactic micro-organisms in a layer of finite depth. J. Fluid Mech. 208, 509-543.

KálnaY DE Rivas, P. 1972 On the use of nonuniform grids in finite-difference equations. J. Comput. Phys. 10, 202-210.

KessLeR, J. O. 1985a Hydrodynamic focusing of motile algal cells. Nature 313, 218-220.

KessleR, J. O. $1985 b$ Co-operative and concentrative phenomena of swimming micro-organisms. Contemp. Phys. 26, 147-166.

Kessler, J. O. 1986 Individual and collective fluid dynamics of swimming cells. J. Fluid Mech. 173, 191-205.

Lennie, T. B., McKenzie, D. P., Moore, D. R. \& Weiss, N. O. 1988 The breakdown of steady convection. J. Fluid Mech. 188, 47-85.

Lister, J. R. 1987 Long wavelength instability of a line plume. J. Fluid Mech. 175, 413-428.

Pedley, T. J. 1988 Bottom-standing plumes in gyrotactic bioconvection. Bull. Am. Phys. Soc. 33, 2282 (Abstr.).

Pedley, T. J., Hill, N. A. \& Kessler, J. O. 1988 The growth of bioconvection patterns in a uniform suspension of gyrotactic micro-organisms. J. Fluid Mech. 195, 223-338.

Pedley, T. J. \& Kessler, J. O. 1987 The orientation of spheroidal micro-organisms swimming in a flow field. Proc. R. Soc. Lond. B 231, 47-70.

Pedley, T. J. \& Kessler, J. O. 1990 A new continuum model for suspensions of gyrotactic micro-organisms. J. Fluid Mech. 212, 155-182.

Pedley, T. J. \& Kessler, J. O. 1992 Hydrodynamic phenomena in suspensions of swimming micro-organisms. Ann. Rev. Fluid Mech. 24, 313-358.

PlatT, J. R. 1961 'Bioconvection patterns' in cultures of free-swimming organisms. Science 133, $1766-1767$.

Plesset, M. S. \& Winet, H. 1974 Bioconvection patterns in swimming micro-organism cultures as an example of Rayleigh-Taylor instability. Nature 248, 441-443.

Roberts, G. O. 1970 Computational meshes for boundary layer problems. In Second Intl Conf. on Numerical Methods in Fluid Dynamics (ed. M. Holt). Lecture Notes in Physics, vol. 8, pp. 171-177. Springer.

Smith, M. K. 1989 The axisymmetric long-wave instability of a concentric two-phase pipe flow. Phys. Fluids. A 1, 494-506.

Tiмm, U. \& Oкuво, A. 1994 Gyrotaxis: A plume model for self-focusing micro-organisms. Bull. Math. Biol. 56, 187-206.

VAHL DAVIS, G. DE 1983 Natural-convection of air in a square cavity - a bench-mark numericalsolution. Intl J. Numer. Meth. Fluids 3, 249-264.

WAGER, H. 1911 On the effect of gravity upon the movements and aggregation of Euglena viridis, Ehrb., and other micro-organisms. Phil. Trans. R. Soc. Lond. B 201, 333-390. 